Ann. Sci. forest., 1969, 26 (2), 225-256.

\title{
LA XYLOCHRONOLOGIE, PERFECTIONNEMENT LOGIQUE DE LA DENDROCHRONOLOGIE
}

\author{
H. POLGE, R. KELLER* \\ Station de Recherches sur la Qualité des Bois, \\ Centre national de Recherches forestières, 54 - Nancy \\ Institut national de la Recherche agronomique
}

\section{SOMMAIRE}

Certaines caractéristiques de structure du bois, lues sur les courbes de variations de la densité, sont plus discriminantes que la largeur des accroissements, pour différencier entre elles les années de production des cernes.

Permettant de mettre en évidence davantage d'années caractéristiques, et d'obtenir des coefficients de coincidence plus nombreux, elles sont, en outre, moins sensibles aux sources de variations autres que le climat.

Une méthode simple de synchronisation, basée sur l'utilisation de profils xylochronologiques synthétiques portant, en abscisse, les largeurs de cernes, et en ordonnée, les densités maximales et minimales annuelles, est par suite proposée.

\section{I. - INTRODUCTION}

La Dendrochronologie (du grec $\delta \varepsilon v \delta \rho o v=$ arbre) peut être définie comme une science de la datation du bois basée sur l'étude des largeurs de cernes, c'est-à-dire sur un critère facilement mesurable du taux annuel de croissance de l'arbre.

Fondée par Douglass (1919 et 1920) il y a près de cinquante ans, elle a connu des développements importants, aussi bien aux Etats-Unis, avec ANTEVs (1925), Schulman (1955), FritTs (1962 et 1965), BanNister (1963), qu'en Nouvelle-Zélande Druce (1966), ou qu'en Europe, avec Huber (1943), Corona (1959), Florence (1962), Pollanschütz (1962), Vinš (1962), Munaut (1966), Serre (1966).

(`) Avec la collaboration technique de S. Garros, G. JAcQuemor et P. Michel qui ont procédé aux enregistrements densitométriques et calculé les pourcentages de coincidence et les années caractéristiques. 
Elle permet maintenant, grâce en particulier à l'utilisation des ordinateurs modernes (FrITTS, 1963) de connaitre rapidement, et avec un seuil de probabilité élevé, les années de production d'une série de cernes annuels, pour peu que I'on dispose d'un assez grand nombre d'échantillons contemporains, susceptibles de fournir une base de référence valable.

La dendrochronologie classique n'utilise cependant qu'une seule des nombreuses données qui peuvent caractériser un accroissement annuel : sa largeur, alors qu'il n'existe, a priori, aucune raison pour que ce soit l'épaisseur radiale cumulée de l'ensemble des cellules produites durant une saison de végétation qui rende le mieux compte du climat qui a régné pendant l'année correspondante.

FritTs (1965) a proposé un schéma très séduisant des liaisons, directes et indirectes, qui peuvent justifier, à partir d'une faible pluviosité et de températures élevées, la production d'un cerne étroit. Mais la plupart des phénomènes physiologiques qu'il met en cause : différenciation des tissus méristématiques, élongation des aiguilles et de la pousse terminale, élaboration d'hormones de croissance, régulation stomatique, transpiration, respiration, assimilation chlorophyllienne, etc., exercent également de façon évidente une action sur la structure interne du bois produit, au même titre d'ailleurs que d'autres facteurs : somme des quantités de chaleur reçue au-dessus d'un scuil de température déterminé au printemps, ou durée de la photo-période à l'automne, qui paraissent régler la longueur de la saison de végétation.

L'épaisseur des membranes cellulaires et le diamètre des lumens, à toutes les étapes, particulièrement au début et à la în, du cycle de croissance, le pourcentage relatif du bois initial et du bois final, la taille et la répartition des vaisseaux, ainsi que la proportion de tissus conducteurs et de tissus de soutien dans les essences feuillues, sont autant de caractéristiques anatomiques qui dépendent étroitement des facteurs de l'environnement, et notamment du climat.

Une caractéristique physique classique permet d'intégrer l'ensemble de ces données plus ou moins hétérogènes : la densité instantanée du bois, qui varie, on le sait, considérablement, non seulement d'un cerne à l'autre, mais encore à l'intérieur même de chacun d'eux, et il n'existe théoriquement aucune raison pour que ce nouveau critère ne soit pas utilisé, au même titre que la largeur des accroissements, pour résoudre les problèmes de datation.

Or, si pendant longtemps on n'a disposé d'aucun moyen pratique pour analyser les variations de la densité du bois, il n'en est plus de même aujourd'hui, puisque l'on peut maintenant obtenir des enregistrements continus de la densité, soit à partir de coupes microscopiques, par photométrie (GREEN et WORRALL, 1964 ; ELLIOTT et BrooK, 1967), ou par absorptiométrie $\beta$ (PHILlıps, 1960), soit à partir de carottes brutes extraites à la tarière sur des arbres vivants, par exploration microdensitométrique de radiographie (PoLGE, 1966) ; ce dernier procédé en particulier est suffisamment rapide et précis pour permettre d'étudier, dans chaque cas, un grand nombre d'échantillons, donc d'obtenir des résultats qui soient statistiquement significatifs.

De nombreuses données peuvent être recueillies sur les courbes de variations de la densité du bois, notamment les densités maximales et minimales annuelles, ainsi que les largeurs des portions de cernes de densité supérieure ou égale à certains 
seuils déterminés, dont l'un peut être choisi de façon telle qu'il corresponde à la densité limite du bois final.

Des techniques nouvelles de datation, basées sur des critères relevant de la structure interne du bois, devraient donc voir le jour maintenant, auxquelles, pour éviter toute confusion, pourrait être donné le nom de Xylochronologie (du grec క̆viov $=$ bois).

\section{II. - RÉSULTATS ANTÉRIEURS}

Dans une étude précédente (PoLge, 1966, op. cit.), nous avons rendu compte du résultat des premières recherches entreprises pour mettre en évidence l'intérêt de certains critères quantitatifs, obtenus sur les courbes de variations de la densité, pour distinguer entre elles les années de production des divers accroissements annuels.

L'expérience portait, rappelons-le, sur deux espèces : Abies grandis et Pseudotsuga menziesii, 50 arbres par espèce, 2 échantillons (Nord et Sud) par arbre, et 11 cernes (1953 à 1963 inclusivement) par échantillon.

La valeur plus ou moins discriminante des diverses caractéristiques relevées (largeur de l'accroissement annuel, largeur du bois d'été, pourcentage de bois d'été, densité maximale annuelle, densité minimale annuelle, et différence entre le maximum de densité d'une année et le minimum de l'année suivante) a été étudiée en utilisant des méthodes classiques d'analyse statistique; les années qui différaient significativement les unes des autres pour les six grandeurs considérées ont été déterminées à l'aide de tests de Duncan et, en outre, des analyses de variance ont permis de calculer, pour les mêmes caractéristiques, et en fonction des diverses sources de variations retenues, le test $F$, c'est-à-dire le rapport de la variance due au traitement à celle de l'erreur, qui est d'autant plus élevé que le critère en cause varie davantage avec le traitement.

Les comparaisons de moyennes ont montré que le nombre d'années qui se distinguaient de façon significative de l'ensemble des autres, était plus élevé pour certaines caractéristiques nouvelles tirées des courbes de variations de la densité (en particulier pour la densité maximale annuelle), que pour la très classique largeur de cerne.

Quant aux résultats des analyses de variance, ils se trouvent résumés dans le tableau ci-dessous qui donne la valeur du test $F$ pour les deux essences étudiées, lorsque l'on prend l'année comme source de variation :

TABLEAU 1

\begin{tabular}{|c|c|c|}
\hline \multirow{2}{*}{ Caractère } & \multicolumn{2}{|c|}{$F$} \\
\hline & Pseudorsuga menziesii & Abies grandis \\
\hline Densité maximale annuelle $\ldots \ldots \ldots$. & 79,07 & 71,83 \\
\hline $\begin{array}{l}\text { Densité maximale année } n \text { - densité mini- } \\
\text { male année } n+1 \ldots \ldots \ldots \ldots \ldots \ldots \ldots\end{array}$ & 57.13 & 80.76 \\
\hline Largeur du cerne .................... & 38,03 & 39,58 \\
\hline Densité minimale annuelle $\ldots . . . \ldots \ldots \ldots$ & 28,67 & 21,87 \\
\hline Pourcentage bois d'été $\ldots . \ldots \ldots \ldots \ldots \ldots$. & 16,36 & 13,51 \\
\hline Largeur bois d'été ..................... & 11,80 & 10,65 \\
\hline
\end{tabular}


On voit que l'ordre des divers critères pour différencier les années est à peu près le même pour les deux essences, et deux caractéristiques nouvelles : la densité maximale annuelle et le contraste de densité de part et d'autre des limites des accroissements, arrivent, dans les deux cas, nettement avant la largeur du cerne, avec une valeur de $F$ qui est, en gros, deux fois plus élevée.

Il était ainsi prouvé pour la première fois que la densité maximale, en particulier, variait considérablement en fonction de l'année de production du cerne considéré, et ceci avait été confirmé par le fait qu'une corrélation étroite avait pu être relevée entre elle et une donnée climatique simple : la pluviosité cumulée des mois d'août, septembre et octobre pour Pseudotsuga menziesii $(r=-0,89$ avec 10 degrés de liberté) et l'insolation cumulée de juillet, août et septembre pour Abies grandis ( $r=$ $+0,81)$.

\section{III. - PREMIÊRE ÉTUDE DE XYLOCHRONOLOGIE PROPREMENT DITE}

\section{1. - Problème posé}

La Station de Recherches sur la Qualité des Bois de Nancy a été amenée incidemment à s'intéresser à des problèmes de datation proprement dite à la demande de Th. KELLER de l'Institut fédéral de Recherches forestières de Zürich-Birmensdorf ; il s'agissait à l'origine d'apprécier les dommages causés à un peuplement d'Epicéas situé dans la plaine alluviale du Rhin par des fumées fluorées émanant d'une usine d'aluminium.

De façon évidente, les vapeurs nocives avaient entraîné une sévère réduction de la largeur de certains cernes, voire, dans quelques cas particuliers, la disparition de tout accroissement ; il convenait donc au départ, pour permettre une évaluation correcte de la perte de production subie, d'affecter à chaque année un cerne donné, en comparant un certain nombre d'arbres du peuplement à des arbres-témoins susceptibles de fournir une base de synchronisation valable.

L'étude a porté en réalité sur 75 arbres provenant, pour 25 , du peuplement le plus endommagé (qui sera désigné ci-après sous le nom de parcelle 1), pour 25, d'un peuplement dont on ignorait au départ s’il avait subi des dégâts notables (parcelle 2), et pour 25, d'un peuplement témoin sûrement indemne (parcelle 3 ) ; en fait, il s'est avéré que le peuplement de la parcelle 3 ne fournissait pas un bon témoin, étant beaucoup plus jeune que celui des parcelles 1 et 2 , mais cet état de choses, qui constitue, à n'en pas douter, un inconvénient sérieux pour l'évaluation de la réduction du rythme de croissance entraînée par les fumées fluorées, peut, à l'inverse, être considéré comme un avantage si l'on veut comparer l'efficacité respective des divers critères pour la datation des accroissements annuels ; il est clair en effet que la meilleure caractéristique sera celle qui réagira le plus aux facteurs du climat, et qui sera, au contraire, la moins sensible aux autres sources de variation : âge et densité du peuplement, interventions humaines, etc.

Les émissions de fumées nocives ayant commencé à être vraiment importantes au cours de la dernière guerre, les caractéristiques de structure des accroissements 
annuels ont été relevées à partir de 1935, de façon à disposer de plusieurs années de croissance normale avant que soit troublé l'équilibre physiologique des arbres en cause ; le nombre total de cernes étudiés se chiffre ainsi à 33 (1935 à 1967 inclusivement) et, pour chacun d'eux, ont été relevées, outre la largeur des cernes, 6 caractéristiques densitométriques : densités maximale et minimale annuelles et largeur des fractions d'accroissement de densité supérieure ou égale à $600,700,800$ et $900 \mathrm{~g} / \mathrm{dm}^{3}$; il ne sera rendu compte ici que des premiers résultats concernant les seules densités maximales et minimales annuelles.

\section{2. - RÉSULTATS}

\subsection{1. - Forme des courbes densitométriques}

Sur les figures 1 à 3 sont regroupés les profils densitométriques de 16 arbres (sur un échantillonnage total de 25) pour chacune des parcelles 1 à 3 respectivement ; les courbes éliminées satisfaisaient toutes autant que les autres aux descriptions qui suivront, le choix ayant été uniquement fait en fonction de la longueur des profils, de façon à faciliter la reproduction photographique de l'ensemble.

En outre, la vitesse moyenne de croissance dans la parcelle 3 étant approximativement double de celle des parcelles 1 et 2 , les enregistrements correspondants ont été faits à une échelle deux fois plus petite afin d'obtenir un format final comparable.

Sous ces réserves, on peut observer, comme ce fut déjà le cas lors de l'étude précitée (Polge, 1966, op. cit.), que les profils de nombreux accroissements ont une forme très caractéristique.

Celui de 1935 se distingue par une silhouette trapue, avec une montée en densité brutale, se traduisant par une pente très rapide à la limite bois initial - bois final ; le profil ne se termine qu'exceptionnellement par une pointe unique très acérée, et l'on observe le plus souvent deux sommets successifs qui, lorsqu'ils sont très rapprochés, arrivent à former un replat, et ceci est vrai, aussi bien pour les arbres à croissance lente des parcelles 1 et 2 , que pour les jeunes arbres, beaucoup plus vigoureux, de la parcelle 3 .

Un autre repère intéressant est fourni par le couple des cernes 1939 et 1940 dont les courbes densitométriques ont généralement des formes extrêmement voisines ; cette remarque s'applique surtout à la parcelle 3 , oủ l'on retrouve des ressemblances frappantes jusque dans les moindres détails (arbres 51 à 54, 56 à 61, 68 et 69); dans les deux autres peuplements, la similitude n'est pas aussi prononcée, mais reste cependant assez remarquable pour de nombreux échantillons, les seules exceptions vraiment notables étant celles des arbres $29,41,42$, et surtout 33 .

Un des accroissements les plus caractéristiques des parcelles 1 et 2 est celui de 1943, c'est-à-dire de la première année qui a suivi l'augmentation des émissions de fumées fluorées durant la dernière guerre ; lorsque ce cerne n'est pas complètement 
absent, il se distingue très nettement des autres par une densité minimale beaucoup plus forte et une densité maximale beaucoup plus faible ; ceci est vrai même lorsque sa largeur est à peu près normale, auquel cas le profil comporte souvent à mi-course un renflement auquel succède une pointe extrêmement fine (arbres 3, 6, 9, 12, 23, $26,29,32,33,38,39,41,42$ ) ; dans la parcelle 3 , on retrouve, à la seule exception de l'arbre 53, cette caractéristique de forme (renflement et pointe terminale), mais maximum et minimum de densité y sont par contre tout à fait normaux.

L'année 1947 se différencie également de la plupart des autres par un maximum de densité très faible, mais d'une part, cette caractéristique est valable aussi bien pour la parcelle 3 que pour les deux autres, et d'autre part, ce maximum de densité est suivi, et non pas précédé, par un minimum exceptionnellement élevé ; en outre, comparé à celui de 1943, le cerne 1947 se distingue en général par une montée en densité plus rapide, et par un replat terminal qui est souvent occasionné par la présence de plusieurs petits pics successifs.

L'énumération de tous les accroissements ayant une forme typique serait trop fastidieuse pour pouvoir être reproduite ici ; on doit cependant mentionner pour terminer le cerne 1960 : son profil est caractérisé par un double sommet dont le premier s'estompe parfois pour ne laisser qu'une trace sous forme d'un renflement plus ou moins marqué ; en outre, la densité minimale de 1960 est très souvent beaucoup plus faible que celle de 1959, et il en est de même pour la densité maximale, généralement inférieure à la fois à celles de l'année précédente et de l'année suivante, ce qui avait déjà été observé pour Abies grandis et Pseudotsuga menziesii à l'occasion de l'étude précitée (Polge, 1966, op. cit.).

\subsection{2. - Pourcentage de coïncidences}

Le calcul des pourcentages de coïncidences fait partie des méthodes classiques de datation qui, appliquées jusqu'ici aux seules largeurs des cernes, peuvent, sans aucune difficulté, l'être maintenant aux données nouvelles tirées des courbes de variations de la densité.

On dit qu'il y a coīncidence pour deux arbres comparés l'un à l'autre lorsque la caractéristique considérée varie dans le même sens, en passant d'un cerne au suivant. Le fait que l'on se réfère chaque fois à l'année précédente fait que, si l'on a relevé les valeurs en cause sur $n$ années, on ne peut calculer les pourcentages de coïncidences que sur un maximum de $n-1$ années.

Quant au nombre de pourcentages que l'on peut obtenir à partir de $m$ arbres pris deux à deux, il est égal, en application des formules de l'analyse combinatoire, à $m(m-1) / 2$.

Dans le cas présent, le nombre de combinaisons s'élève donc à 300 pour chacune des parcelles et pour chaque critère étudié (largeur de cernes, densité maximale et densité minimale annuelles), soit un total de 2700 pourcentages de coïncidences qui sont consignés en Annexe sous les numéros 1 à 9.

Le nombre d'années sur lesquelles portent les comparaisons se chiffre, en règle 
générale, à 32, mais il est parfois inférieur en raison de l'absence de données pour quelques accroissements.

Sur les tableaux qui figurent en annexe, sont soulignés les rapports de coïncidences supérieurs ou égaux à $75 \%$; ce seuil de « signification » relativement peu élevé a été choisi pour tenir compte du fait que les années oủ il y a égalité avec l'année précédente pour un des arbres, ont été prises en compte dans le nombre total de comparaisons, alors qu'il n'existe qu'une très faible probabilité pour que l'on ait aussi une égalité absolue entre les mêmes années pour tous les autres arbres de l'expérience, en raison de la grande précision des mesures, aussi bien de largeurs de cernes que de densités.

Le nombre des comparaisons où il y a coïncidence pour au moins $75 \%$ des années est reporté dans le tableau suivant :

\section{TABLEAU 2}

Nombre de pourcentages de coincidences supérieurs ou égaux à $75 \%$

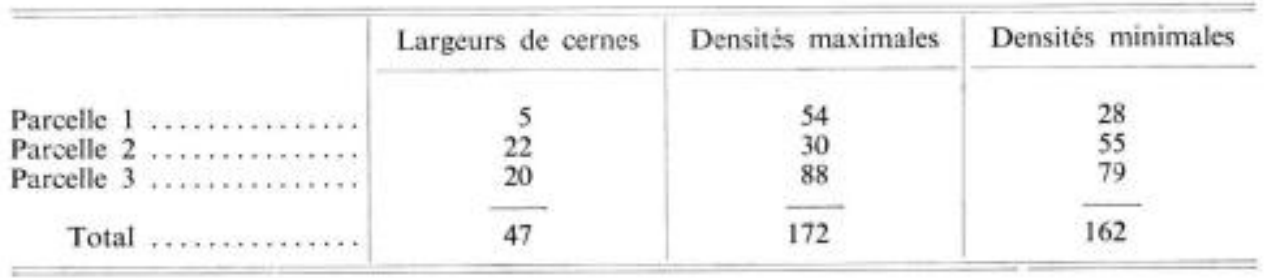

On peut faire les constatations suivantes :

- quelle que soit la parcelle, le nombre de pourcentages de coïncidences supérieurs ou égaux à $75 \%$ est nettement plus faible pour les largeurs de cernes que pour les densités maximales ou les densités minimales, ce qui confirme bien que ces dernières caractéristiques sont plus discriminantes pour différencier entre elles les années de production des cernes;

- d'une façon générale, les coïncidences sont meilleures dans le peuplement témoin, en raison sans doute du fait que la sensibilité aux fumées fluorées dans les deux autres parcelles a varié considérablement suivant les individus, d'oủ une plus grande dispersion des résultats ;

- enfin, il convient de noter les informations extrêmement intéressantes obtenues sur les densités maximales et minimales dans la parcelle 3 , où respectivement 88 et 79 comparaisons sur 300 (soit 29 et $26 \%$ du total) ont conduit à des pourcentages de coỉncidences supérieurs ou égaux à $75 \%$.

\subsection{3. - Années caractéristiques}

Au lieu de s'intéresser comme précédemment au nombre d'années où il $\mathrm{y}$ a coïncidence pour l'ensemble des arbres comparés deux à deux, on peut tout aussi bien calculer celui des individus ayant, pour chaque année prise isolément, un même 
sens de variation, en le rapportant, soit au nombre total d'arbres, soit seulement, comme ce fut le cas pour cette étude, à celui des sujets pour lesquels il y a eu effectivement variation de la caractéristique en cause, c'est-à-dire cas d'égalité déduits.

Lorsque le rapport ainsi formé atteint ou dépasse un certain seuil (on a choisi ici celui de $80 \%$, on considère que l'on a affaire à une année caractéristique.

Dans les tableaux ci-dessous, on a indiqué, pour chaque critère étudié et pour chaque parcelle, le nombre total net d'individus (données manquantes et cas d'égalité non compris), et le nombre de ceux pour lesquels la caractéristique en cause a, soit augmenté, soit diminué, par rapport à l'année précédente ; sont à nouveau indiqués en italique les cas où le seuil susmentionné est atteint ou dépassé.

\section{TABLEAU 3}

Années caractéristiques. Parcelle $I$

\begin{tabular}{|c|c|c|c|c|c|c|c|c|c|c|}
\hline & & \multicolumn{3}{|c|}{$\begin{array}{l}\text { Largeurs de cernes } \\
\text { Nombre d'arbres }\end{array}$} & \multicolumn{3}{|c|}{$\begin{array}{c}\text { Densités maximales } \\
\text { Nombre d'arbres }\end{array}$} & \multicolumn{3}{|c|}{$\begin{array}{l}\text { Densités minimales } \\
\text { Nombre d'arbres }\end{array}$} \\
\hline & & $\begin{array}{c}\text { Total } \\
\text { net }\end{array}$ & $\begin{array}{c}\text { En } \\
\text { augmen- } \\
\text { tation }\end{array}$ & $\begin{array}{c}\text { En } \\
\text { dimi- } \\
\text { nution }\end{array}$ & $\begin{array}{c}\text { Total } \\
\text { net }\end{array}$ & $\begin{array}{l}\text { En } \\
\text { augmen- } \\
\text { tation }\end{array}$ & $\begin{array}{c}\text { En } \\
\text { dimi- } \\
\text { nution }\end{array}$ & $\begin{array}{c}\text { Total } \\
\text { net }\end{array}$ & $\begin{array}{l}\text { En } \\
\text { augmen- } \\
\text { tation }\end{array}$ & $\begin{array}{c}\text { En } \\
\text { dimi- } \\
\text { nution }\end{array}$ \\
\hline 1936 & $\ldots \ldots \ldots$ & 23 & 13 & 10 & 25 & 3 & 22 & 23 & 22 & 1 \\
\hline 1937 & ,....... & 24 & 3 & $2 l$ & 25 & $2 I$ & 4 & 25 & 3 & 22 \\
\hline 1938 & $\ldots \ldots \ldots$ & 24 & 8 & 16 & 20 & 16 & 4 & 23 & 23 & 0 \\
\hline 1939 & $\ldots \ldots$ & 25 & 16 & 9 & 25 & 0 & 25 & 22 & 18 & 4 \\
\hline 1940 & $\ldots \ldots \ldots$ & 25 & 18 & 7 & 25 & 18 & 7 & 25 & 3 & 22 \\
\hline 1941 & $\ldots \ldots \ldots$ & 25 & 18 & 7 & 25 & 22 & 3 & 23 & 6 & 17 \\
\hline 1942 & $\ldots \ldots \ldots$ & 25 & 1 & 24 & 23 & 16 & 7 & 23 & 9 & 14 \\
\hline 1943 & $\ldots \ldots \ldots$ & 25 & 0 & 25 & 16 & 0 & 16 & 18 & 18 & 0 \\
\hline 1944 & $\ldots \ldots \ldots$ & 19 & 8 & 11 & 14 & 13 & 1 & 14 & 7 & 7 \\
\hline 1945 & $\ldots \ldots \ldots$ & 22 & 21 & 1 & 20 & 20 & 0 & 18 & 6 & 12 \\
\hline 1946 & $\ldots+\ldots$ & 24 & 23 & 1 & 23 & 14 & 9 & 22 & 2 & 20 \\
\hline 1947 & $\ldots \ldots \ldots$ & 25 & 9 & 16 & 25 & 0 & 25 & 21 & 1 & 20 \\
\hline 1949 & ......... & 25 & 9 & 16 & 25 & 25 & 0 & 24 & 23 & 1 \\
\hline 1949 & $\ldots \ldots \ldots$ & 25 & 15 & 10 & 25 & 15 & 10 & 24 & 1 & 23 \\
\hline 1950 & $\ldots \ldots \ldots$ & 24 & 6 & 18 & 25 & 8 & 17 & 23 & 14 & 9 \\
\hline 1951 & ......... & 25 & 7 & 18 & 25 & 7 & 18 & 22 & 6 & 16 \\
\hline 1952 & ......... & 24 & 7 & 17 & 23 & 17 & 6 & 22 & 21 & 1 \\
\hline 1953 & ......... & 23 & 8 & 15 & 22 & 8 & 14 & 23 & 18 & 5 \\
\hline 1954 & $\ldots \ldots \ldots$ & 20 & 14 & 6 & 20 & 4 & 16 & 21 & 4 & 17 \\
\hline 1955 & $\ldots \ldots \ldots$ & 21 & 9 & 12 & 21 & 16 & 5 & 19 & 5 & 14 \\
\hline 1956 & $\ldots . .$. & 21 & 5 & 16 & 20 & 10 & 10 & 20 & 17 & 3 \\
\hline 1957 & $\ldots \ldots \ldots$ & 22 & 10 & 12 & 18 & 12 & 6 & 18 & 10 & 8 \\
\hline 1958 & $\ldots \ldots \ldots$ & 20 & 12 & 8 & 20 & 10 & 10 & 18 & 9 & 9 \\
\hline 1959 & .......... & 23 & 17 & 6 & 21 & 17 & 4 & 23 & 15 & 8 \\
\hline 1960 & $\ldots \ldots \ldots$ & 23 & 19 & 4 & 23 & 4 & 19 & 23 & 1 & 22 \\
\hline 1961 & $\ldots \ldots \ldots$ & 22 & 16 & 6 & 23 & 19 & 4 & 20 & 17 & 3 \\
\hline 1962 & $\ldots \ldots \ldots$ & 20 & 3 & 17 & 24 & 9 & 15 & 21 & 9 & 12 \\
\hline 1963 & ......... & 24 & 10 & 14 & 24 & 16 & 8 & 23 & 13 & 10 \\
\hline 1964 & ......... & 24 & 22 & 2 & 21 & 9 & 12 & 22 & 3 & 19 \\
\hline 1965 & $\ldots \ldots$. & 24 & 11 & 13 & 22 & 1 & $2 I$ & 18 & 7 & 11 \\
\hline 1966 & $\ldots \ldots \ldots$ & 22 & 14 & 8 & 21 & 17 & 4 & 22 & 16 & 6 \\
\hline 1967 & $\ldots \ldots \ldots$ & 20 & 2 & 18 & 17 & 11 & 6 & 20 & 13 & 7 \\
\hline
\end{tabular}




\section{TABLEAU 4}

Années caractéristiques. Parcelle 2

\begin{tabular}{|c|c|c|c|c|c|c|c|c|c|c|}
\hline & & \multicolumn{3}{|c|}{$\begin{array}{l}\text { Largeurs de cernes } \\
\text { Nombre d'arbres }\end{array}$} & \multicolumn{3}{|c|}{$\begin{array}{l}\text { Densités maximales } \\
\text { Nombre d'arbres }\end{array}$} & \multicolumn{3}{|c|}{$\begin{array}{l}\text { Densités minimales } \\
\text { Nombre d'arbres }\end{array}$} \\
\hline & & $\begin{array}{c}\text { Total } \\
\text { net }\end{array}$ & $\begin{array}{c}\text { En } \\
\text { augmen- } \\
\text { tation }\end{array}$ & $\begin{array}{c}\text { En } \\
\text { dimi- } \\
\text { nution }\end{array}$ & $\begin{array}{c}\text { Total } \\
\text { net }\end{array}$ & $\begin{array}{c}\text { En } \\
\text { augmen- } \\
\text { tation }\end{array}$ & $\begin{array}{c}\text { En } \\
\text { dimi- } \\
\text { nution }\end{array}$ & $\begin{array}{c}\text { Total } \\
\text { net }\end{array}$ & $\begin{array}{c}\text { En } \\
\text { augmen- } \\
\text { tation }\end{array}$ & $\begin{array}{c}\text { En } \\
\text { dimi- } \\
\text { nution }\end{array}$ \\
\hline 1936 & $\ldots \ldots \ldots$ & 24 & 7 & 17 & 24 & 3 & $2 l$ & 25 & 23 & 2 \\
\hline 1937 & , n...... & 25 & 4 & 21 & 24 & 15 & 9 & 23 & 3 & 20 \\
\hline 1938 & ......... & 24 & 3 & 21 & 23 & 13 & 10 & 22 & 17 & 5 \\
\hline 1939 & ........ & 24 & 18 & 6 & 24 & 3 & 21 & 25 & 20 & 5 \\
\hline 1940 & ......... & 25 & 12 & 13 & 24 & 12 & 12 & 23 & 5 & 18 \\
\hline 1941 & ........ & 25 & 16 & 9 & 25 & 22 & 3 & 18 & 6 & 12 \\
\hline 1942 & ........ & 25 & 8 & 17 & 25 & 16 & 9 & 22 & 10 & 12 \\
\hline 1943 & $\ldots \ldots \ldots$ & 24 & 1 & 23 & 17 & 1 & 16 & 20 & 19 & 1 \\
\hline 1944 & ......... & 16 & 2 & 14 & 16 & 15 & 1 & 15 & 10 & 5 \\
\hline 1945 & ........ & 24 & 23 & 1 & 18 & 18 & 0 & 14 & 2 & 12 \\
\hline 1946 & ......... & 25 & 25 & 0 & 21 & 13 & 8 & 23 & $i$ & 22 \\
\hline 1947 & $\ldots \ldots \ldots$ & 25 & 10 & 15 & 24 & 1 & 23 & 24 & i & 23 \\
\hline 1948 & $\ldots \ldots \ldots$ & 22 & 9 & 13 & 25 & 24 & 1 & 25 & 24 & 1 \\
\hline 1949 & ........ & 25 & 14 & 11 & 22 & 12 & 10 & 25 & 1 & 24 \\
\hline 1950 & .......... & 22 & 9 & 13 & 24 & 9 & is & 21 & 15 & 6 \\
\hline 1951 & ......... & 24 & 1 & 23 & 24 & 2 & 22 & 24 & 3 & 21 \\
\hline 1952 & ......... & 24 & 14 & 10 & 24 & 17 & 7 & 25 & 23 & 2 \\
\hline 1953 & ........... & 23 & 14 & 9 & 23 & 14 & 9 & 21 & 16 & 5 \\
\hline 1954 & ........... & 25 & 20 & 5 & 25 & 1 & 24 & 22 & 4 & 18 \\
\hline 1955 & ........ & 25 & 16 & 9 & 23 & 18 & 5 & 21 & 5 & 16 \\
\hline 1956 & ......... & 24 & 3 & 27 & 24 & 9 & 15 & 21 & 9 & 12 \\
\hline 1957 & ......... & 24 & 15 & 9 & 24 & 20 & 4 & 23 & 16 & 7 \\
\hline 1958 & $\ldots \ldots \ldots$ & 25 & 17 & 8 & 22 & 7 & 15 & 24 & 4 & 20 \\
\hline 1959 & ......... & 25 & 3 & 22 & 24 & 18 & 6 & 25 & 24 & I \\
\hline 1960 & ......... & 24 & 16 & 8 & 25 & 3 & 22 & 24 & 1 & 23 \\
\hline 1961 & .......... & 24 & 19 & 5 & 23 & 21 & 2 & 24 & 19 & 5 \\
\hline 1962 & ......... & 25 & 1 & 24 & 23 & 3 & 20 & 20 & 5 & 15 \\
\hline 1963 & ......... & 25 & 6 & 19 & 25 & 20 & 5 & 25 & 23 & 2 \\
\hline 1964 & ,......... & 25 & 21 & 4 & 23 & 7 & 16 & 25 & 2 & 23 \\
\hline 1965 & ........... & 24 & 16 & 8 & 24 & 5 & 19 & 24 & 16 & 8 \\
\hline 1966 & ......... & 23 & 9 & 14 & 25 & 10 & 15 & 20 & 9 & 11 \\
\hline 1967 & $\ldots \ldots$ & 21 & 3 & 18 & 18 & 10 & 8 & 21 & 16 & 5 \\
\hline
\end{tabular}

En outre, sur le tableau 6 sont regroupés les accroissements annuels caractéristiques au point de vue largeurs de cernes, densités maximales et densités minimales, non seulement pour chaque parcelle prise isolément, mais encore pour l'ensemble des trois peuplements étudiés, les lettres $\mathrm{M}$ et $\mathrm{m}$ désignant les années oủ le critère en cause a été respectivement plus élevé et moins élevé que l'année précédente, au seuil de signification de $80 \%$.

On voit que la même tendance générale se dégage de cette nouvelle comparaison, à savoir la meilleure homogénéité des résultats pour les critères de densité que pour les largeurs de cernes.

Si l'on fait le total des années caractéristiques pour les trois parcelles étudiées séparément, la supériorité des densités maximales et minimales, bien que notable, 


\section{TABLEAU 5}

Années caractéristiques. Parcelle 3

\begin{tabular}{|c|c|c|c|c|c|c|c|c|c|c|}
\hline & & \multicolumn{3}{|c|}{$\begin{array}{l}\text { Largeurs de cernes } \\
\text { Nombre d'arbres }\end{array}$} & \multicolumn{3}{|c|}{$\begin{array}{c}\text { Densités maximales } \\
\text { Nombre d'arbres }\end{array}$} & \multicolumn{3}{|c|}{$\begin{array}{c}\text { Densités minimales } \\
\text { Nombre d'arbres }\end{array}$} \\
\hline & & $\begin{array}{c}\text { Total } \\
\text { net }\end{array}$ & $\begin{array}{c}\text { En } \\
\text { augmen- } \\
\text { tation }\end{array}$ & $\begin{array}{c}\text { En } \\
\text { dimi- } \\
\text { nution }\end{array}$ & $\begin{array}{c}\text { Total } \\
\text { net }\end{array}$ & $\begin{array}{c}\text { En } \\
\text { augmen- } \\
\text { tation }\end{array}$ & $\begin{array}{c}\text { En } \\
\text { dimi- } \\
\text { nution }\end{array}$ & $\begin{array}{c}\text { Total } \\
\text { net }\end{array}$ & $\begin{array}{c}\text { En } \\
\text { augmen- } \\
\text { tation }\end{array}$ & $\begin{array}{c}\text { En } \\
\text { dimi- } \\
\text { nution }\end{array}$ \\
\hline 1936 & $\ldots \ldots \ldots$ & 25 & 22 & 3 & 25 & 1 & 24 & 25 & 25 & 0 \\
\hline 1937 & n...... & 25 & 9 & 16 & 25 & 22 & 3 & 22 & 2 & 20 \\
\hline 1938 & ......... & 25 & 14 & 11 & 24 & 11 & 13 & 22 & 13 & 9 \\
\hline 1939 & n. $\ldots$ & 25 & 23 & 2 & 25 & 0 & 25 & 20 & 6 & 14 \\
\hline 1940 & , . . . . & 24 & 18 & 6 & 25 & 9 & 16 & 19 & 13 & 6 \\
\hline 1941 & ........ & 25 & 0 & 25 & 24 & 21 & 3 & 22 & 5 & 17 \\
\hline 1942 & . $\ldots \ldots \ldots$ & 25 & 18 & 7 & 25 & 25 & 0 & 19 & 15 & 4 \\
\hline 1943 & , n., & 25 & 2 & 23 & 25 & 20 & 5 & 25 & 22 & 3 \\
\hline 1944 & ......... & 23 & 1 & 22 & 25 & 5 & 20 & 24 & 23 & 1 \\
\hline 1945 & ......... & 23 & 4 & 19 & 24 & 23 & 1 & 24 & 1 & 23 \\
\hline 1946 & ......... & 24 & 10 & 14 & 24 & 18 & 6 & 24 & 23 & 1 \\
\hline 1947 & $\ldots \ldots$, & 25 & 1 & 24 & 25 & 0 & 25 & 25 & 0 & 25 \\
\hline 1948 & n...... & 25 & 23 & 2 & 25 & 25 & 0 & 25 & 25 & 0 \\
\hline 1949 & ........ & 24 & 8 & 16 & 25 & 17 & 8 & 25 & 0 & 25 \\
\hline 1950 & ........ & 25 & 20 & 5 & 24 & 10 & 14 & 21 & 13 & 8 \\
\hline 1951 & ......... & 25 & 11 & 14 & 25 & 5 & 20 & 20 & 6 & 14 \\
\hline 1952 & ........ & 25 & 7 & 18 & 25 & $2 I$ & 4 & 18 & 16 & 2 \\
\hline 1953 & ......... & 25 & 23 & 2 & 24 & 7 & 17 & 25 & 24 & 1 \\
\hline 1954 & ........ & 25 & 10 & 15 & 25 & 1 & 24 & 24 & 1 & 23 \\
\hline 1955 & ........ & 23 & 9 & 14 & 24 & 16 & 8 & 24 & 2 & 22 \\
\hline 1956 & $\ldots, \ldots$, & 25 & 16 & 9 & 25 & 4 & $2 I$ & 24 & 17 & 7 \\
\hline 1957 & ......... & 24 & 17 & 7 & 24 & $2 I$ & 3 & 25 & 22 & 3 \\
\hline 1958 & $\ldots \ldots \ldots$ & 25 & 5 & 20 & 24 & 17 & 7 & 25 & 2 & 23 \\
\hline 1959 & ......... & 23 & 5 & 18 & 23 & 15 & 8 & 24 & 24 & 0 \\
\hline 1960 & ......... & 24 & 10 & 14 & 25 & 1 & 24 & 23 & 4 & 19 \\
\hline 1961 & ........ & 25 & 7 & 18 & 25 & 22 & 3 & 23 & 20 & 3 \\
\hline 1962 & , n...... & 24 & 5 & 19 & 24 & 14 & 10 & 21 & 3 & 18 \\
\hline 1963 & .......... & 25 & 9 & 16 & 22 & 15 & 7 & 22 & 22 & 0 \\
\hline 1964 & ........ & 25 & 18 & 7 & 22 & 13 & 9 & 24 & 1 & 23 \\
\hline 1965 & ,........ & 23 & 10 & 13 & 25 & 2 & 23 & 21 & 12 & 9 \\
\hline 1966 & .......... & 25 & 3 & 22 & 24 & 15 & 9 & 22 & 20 & 2 \\
\hline 1967 & .......... & 8 & 6 & 2 & 10 & 6 & 4 & 22 & 13 & 9 \\
\hline
\end{tabular}

n'est pas aveuglante, mais elle le devient lorsque l'on regroupe l'ensemble des 75 arbres de l'échantillonnage, qu'ils proviennent, ou non, des peuplements fluorés : dans ce cas, les années caractéristiques au point de vue largeurs de cernes ne sont plus qu'au nombre de 4 , mais le chiffre est triple pour la densité maximale, et quadruple pour la densité minimale.

L'intérêt de ce dernier critère doit être noté, car jusqu'ici, et en particulier dans l'étude précitée relative à Abies grandis et Pseudotsuga menziesii, les densités maximales avaient paru bien supérieures pour différencier les années de production des cernes successifs ; or ici, c'est, de façon très nette, la densité minimale qui fournit le plus d'années caractéristiques, quel que soit le mode de comparaison utilisé. 


\section{TABLEAU 6}

Années caractéristiques

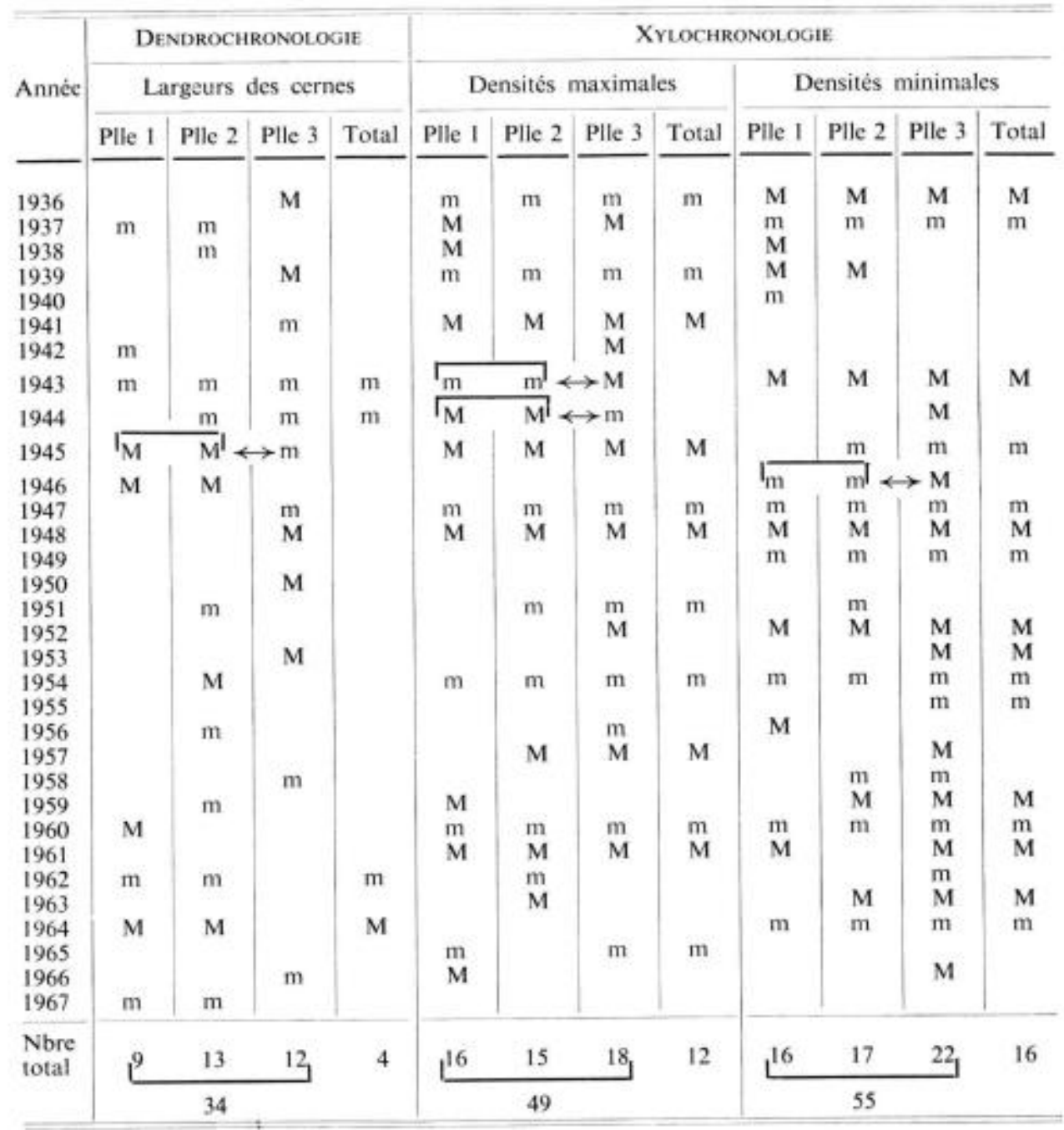

Dernière remarque concernant le tableau 6 : on y a souligné, à l'aide d'une accolade horizontale suivie d'une flèche, les cas où le sens de variation n'est pas le même selon les parcelles; cela ne se produit jamais entre les peuplements 1 et 2 , mais 4 fois entre le groupe des parcelles 1 et 2 d'une part, et 3 d'autre part, et les années intéressées sont précisément les 4 années qui ont suivi l'aggravation des émissions fluorées. Il est certain que le schéma normal de variation est celui de la parcelle 3, et que, pour les autres, il suffit qu'il y ait une année une réaction très brutale dans un sens pour qu'une évolution en sens contraire apparaisse l'année sui- 
vante, pour peu qu'une tendance à la normalisation se fasse jour, puisque les années caractéristiques sont toujours déterminées par référence à l'accroissement précédent ; ainsi s'expliquent les oppositions constatées en 1943, 1944 et 1945 ; celle observée en 1946 en ce qui concerne les densités minimales est beaucoup plus étonnante, et aucune explication valable ne peut être proposée à ce sujet.

\subsection{4. - Influence des facteurs autres que le climat}

Les facteurs climatiques sont bien entendu ceux qui interviennent au premier chef pour expliquer les variations de structure des accroissements d'une année à l'autre. Ce ne sont cependant pas les seuls, puisque, d'une part, il existe des gradients généraux de variations avec l'âge (en gros : diminution des largeurs de cernes, augmentation des densités), et que, d'autre part, des influences extérieures peuvent se faire sentir : influences humaines (éclaircie, élagage, fumées industrielles), ou autres (dégâts d'insectes par exemple) ; enfin, il ne faut pas oublier les effets du patrimoine héréditaire qui font que, toutes choses égales par ailleurs, deux individus de même âge, placés dans le même environnement, peuvent produire des accroissements de structure très différente à tous points de vue.

Dans ces conditions, il était intéressant d'utiliser les résultats de la présente expérience pour essayer de voir comment les trois critères étudiés variaient en fonction des facteurs autres que climatiques : âge et fumées fluorées dans le cas particulier.

\subsection{1. - Observations sur les courbes représentatives des moyennes de parcelles}

Ces courbes sont reportées sur la figure 4 .

On constate immédiatement que, pour toutes les caractéristiques, les résultats concernant les parcelles 1 et 2 sont extrêmement voisins, et s'écartent les uns et les autres très nettement de ceux de la parcelle 3 ; mais les différences qui sont très importantes pour les largeurs de cernes semblent bien moindres pour les densités maximales, et encore plus faibles pour les densités minimales.

L'influence de l'âge est très sensible jusque vers 1941, avec des cernes plus larges et des maximums de densité moins élevés dans la parcelle 3 que dans les deux autres ; on peut penser qu'à cette époque le peuplement témoin est passé de l'état juvénile à l'état adulte, ce qui entraine, on le sait, des changements considérables dans la structure du bois ; par la suite, la vitesse radiale de croissance y demeure plus rapide que dans les parcelles fluorées, mais les écarts au point de vue densité maximale et densité minimale deviennent très faibles.

La comparaison des accroissements 1943 et 1947, déjà évoqué au paragraphe 3.21 , est très instructive : pour le premier, il y a opposition complète entre les courbes des parcelles 1 et 2 d'une part, et de la parcelle 3 d'autre part, avec notamment un cerne plus large, un minimum de densité plus faible, et surtout une densité maximale qui passe par un sommet, alors que celles des parcelles 1 et 2 sont, au contraire, exceptionnellement peu élevées.

En 1947 par contre, il y a presque identité absolue entre les divers peuplements pour les trois critères; le phénomène est tellement net que l'on peut en déduire, 


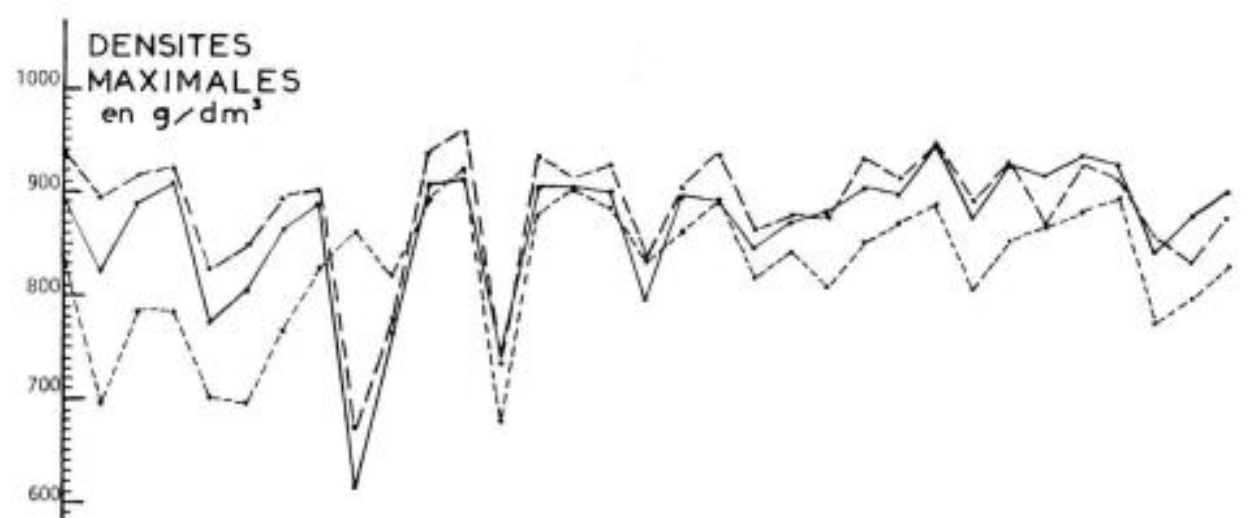

DENSITES
MINIMALES
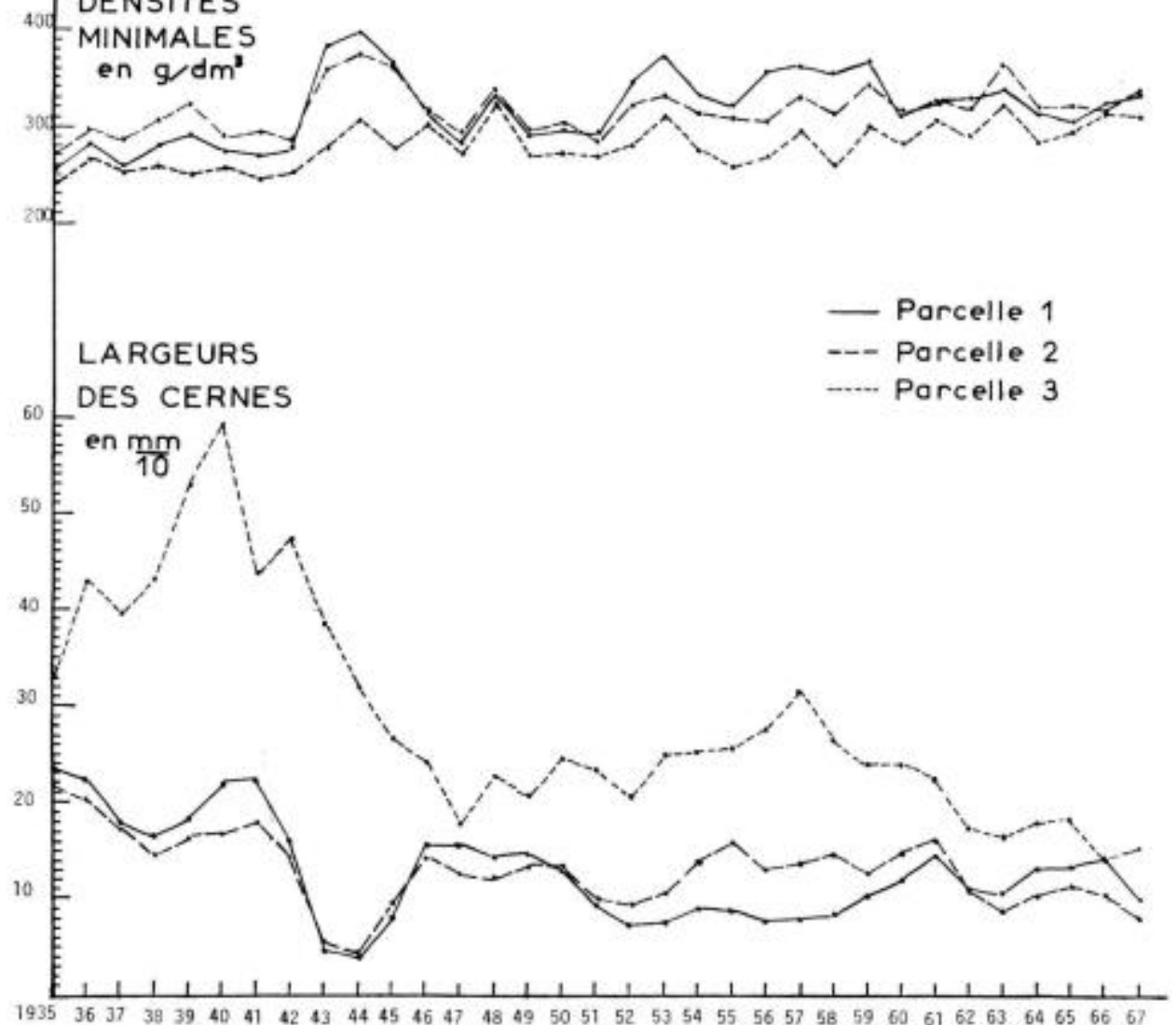
sans risque d'erreur, que la très faible densité maximale de 1943 a bien été occasionnée par l'émission brutale d'une grande quantité de fumées nocives, alors que celle de 1947 ne serait liée qu'aux facteurs du climat.

\subsection{2. - Coefficients de variations entre les moyennes de parcelles}

La dispersion plus ou moins grande des courbes de la figure 4 est certes intéressante à étudier, mais elle dépend naturellement des échelles adoptées pour les diverses caractéristiques ; bien que celles qui ont été choisies soient en rapport avec les valeurs moyennes des trois critères analysés, il est préférable, pour avoir une idée précise des écarts entre parcelles, de recourir à une expression mathématique homogène de la dispersion, à savoir le coefficient de variation (rapport de l'écart-type à la moyenne).

Le tableau 7 donne les valeurs de ce coefficient pour les 33 années de l'expérience et pour les trois caractéristiques étudiées.

Tableau 7

Coefficients de variation entre les moyennes des parcelles

\begin{tabular}{|c|c|c|c|}
\hline Années & Largeurs de cernes & Densités maximales & Densités minimales \\
\hline $1935 \ldots \ldots \ldots \ldots \ldots$ & 23,07 & 5,99 & 6,06 \\
\hline $1936 \ldots \ldots \ldots \ldots$ & 43,77 & 12,70 & 5,13 \\
\hline $1937 \ldots \ldots \ldots \ldots \ldots$ & 51,12 & 7,98 & 7,25 \\
\hline $1938 \ldots \ldots \ldots \ldots \ldots$ & 64,12 & 8,73 & 8,35 \\
\hline $1939 \ldots \ldots \ldots \ldots$ & 69,84 & 8,19 & 12,70 \\
\hline $1940 \ldots \ldots \ldots \ldots \ldots$ & 70,71 & 9,98 & 5,86 \\
\hline $1941 \ldots \ldots \ldots \ldots \ldots$ & 48,33 & 7,99 & 8,92 \\
\hline $1942 \ldots \ldots \ldots \ldots \ldots$ & 70,91 & 4,67 & 6,34 \\
\hline $1943 \ldots \ldots \ldots \ldots \ldots$ & 117,38 & 18,05 & 15,90 \\
\hline $1944 \ldots \ldots \ldots \ldots \ldots$ & 119,39 & 3,75 & 13,20 \\
\hline $1945 \ldots \ldots \ldots \ldots \ldots$ & 69,94 & 2,59 & 15,37 \\
\hline $1946 \ldots . . . . . . . .$. & 28,39 & 2,51 & 1,96 \\
\hline $1947 \ldots \ldots \ldots \ldots \ldots$ & 16,16 & 4,95 & 4,23 \\
\hline $1948 \ldots \ldots \ldots \ldots \ldots$ & 34,08 & 3,20 & 1,86 \\
\hline $1949 \ldots \ldots \ldots \ldots \ldots$ & 24,14 & 0,73 & 4,69 \\
\hline $1950 \ldots \ldots \ldots \ldots \ldots$ & 38,72 & 2,37 & 5,74 \\
\hline $1951 \ldots \ldots \ldots \ldots$ & 54.91 & 3,02 & 4,21 \\
\hline $1952 \ldots \ldots \ldots \ldots \ldots$ & 55,96 & 0,39 & 10,62 \\
\hline $1953 \ldots \ldots \ldots \ldots$ & 63,49 & 2,86 & 9,28 \\
\hline $1954 \ldots \ldots \ldots \ldots \ldots$ & 50,79 & 2,64 & 9,61 \\
\hline $1955 \ldots \ldots \ldots \ldots$ & 49,96 & 2,24 & 11,60 \\
\hline $1956 \ldots \ldots \ldots \ldots \ldots$ & 62,15 & 4,74 & 14,43 \\
\hline $1957 \ldots \ldots \ldots \ldots \ldots$ & 68,12 & 4,69 & 11,05 \\
\hline $1958 \ldots \ldots \ldots \ldots \ldots$ & 54,36 & 2,48 & 15,94 \\
\hline $1959 \ldots \ldots \ldots \ldots \ldots$ & 45,96 & 3,63 & 10,35 \\
\hline $1960 \ldots \ldots \ldots \ldots$ & 36,31 & 5,12 & 5,45 \\
\hline $1961 \ldots \ldots \ldots \ldots \ldots$ & 22,65 & 4,87 & 3,76 \\
\hline $1962 \ldots \ldots \ldots \ldots \ldots$ & 27,56 & 3,19 & 6,49 \\
\hline $1963 \ldots \ldots \ldots \ldots \ldots$ & 32,79 & 3,33 & 6,66 \\
\hline $1964 \ldots \ldots \ldots \ldots \ldots$ & 26,50 & 1,87 & 6,00 \\
\hline $1965 \ldots \ldots \ldots \ldots \ldots$ & 24,10 & 5,41 & 4,39 \\
\hline 1966 ............... & 16,31 & 4,84 & 1,42 \\
\hline 1967 ............ & 33,00 & 4,25 & 4,56 \\
\hline Total ......... & 1614,99 & 163,95 & 259,38 \\
\hline
\end{tabular}


Il se confirme que la variabilité des largeurs de cernes est beaucoup plus importante que celle des maxima et des minima de densité (avec un coefficient moyen de variation respectivement 10 fois et 6 fois plus élevé) ; ceci n'est pas seulement lié aux différences entre les parcelles 1 et 2 d'une part, et la parcelle 3 de l'autre pendant la période de croissance juvénile de ce dernier peuplement, puisque le même écart subsiste entre les coefficients de variation pour les tout derniers accroissements produits.

\subsection{3. - Corrélations entre les moyennes de parcelles}

Les coefficients de corrélation entre parcelles sont regroupés sur le tableau 8.

TABLEAU 8

Coefficients de corrélation entre les moyennes de parcelles

\begin{tabular}{l|c|c|c}
\hline & Largeurs des cernes & Densités maximales & Dinsités minimales \\
\hline Entre parcelles 1 et $2 \ldots$ & $0,78^{* *}$ & $0,91^{* *}$ & $0,82^{* *}$ \\
\hline Entre parcelles 1 et $3 \ldots$ & $0,36^{* s}$ & $0,49^{*}$ & $0,60^{* *}$ \\
\hline Entre parcelles 2 et $3 \ldots$ & $0,45^{*}$ & $0,46^{*}$ & $0,69^{* *}$ \\
\hline
\end{tabular}

On voit qu'une corrélation extrêmement étroite existe entre les résultats des parcelles 1 et 2 , quelle que soit la caractéristique en cause, ce qui, ajouté à toutes les remarques qui ont pu être faites précédemment, laisse supposer que le peuplement 2 a bien été soumis aux mêmes phénomènes extérieurs que le premier ; mais on constate surtout que la largeur des cernes fournit encore une fois le critère le moins intéressant, les coefficients de corrélation la concernant étant toujours les plus faibles, que l'on compare entre elles les parcelles fluorées, ou que la comparaison soit faite entre l'une ou l'autre d'entre elles et la parcelle témoin.

La densité minimale, par contre, apparaît à nouveau comme un excellent critère de datation, puisqu'elle arrive deux fois en tête, et qu'elle n'a, pour la troisième comparaison, qu'un retard très faible sur la densité maximale ; le minimum de densité annuelle semble donc, dans l'ensemble, à la fois très lié aux facteurs du climat, et très peu sensible aux autres sources de variations, ce qui constitue en l'occurrence un double avantage.

\section{3. - APPORT DE LA XYLOCHRONOLOGIE A LA DENDROCHRONOLOGIE}

Les résultats précédents ont montré l'intérêt de certains critères tirés des courbes de variations de la densité du bois pour différencier les années de production des 
cernes ; encore convient-il de savoir si les renseignements apportés par les densités maximales et minimales annuelles s'ajoutent à ceux déjà donnés par les largeurs de cernes, ou s'ils font en quelque sorte double emploi avec eux. Ceci pose le problème des liaisons éventuelles entre caractéristiques auxométriques et caractéristiques densitométriques, auquel une réponse partielle peut être fournie par l'étude des coefficients de corrélation entre les trois critères étudiés qui font l'objet du tableau 9 :

\section{TABleaU 9}

Coefficients de corrélation entre caractères

\begin{tabular}{|c|c|c|c|c|}
\hline & $\begin{array}{l}\text { Parcelle I } \\
\text { (24 DL) }\end{array}$ & $\begin{array}{l}\text { Parcelle } 2 \\
\text { (24 DL) }\end{array}$ & $\begin{array}{l}\text { Parcelle }{ }^{3} \\
\text { (24 DL) }\end{array}$ & $\begin{array}{l}\text { Ensemble } \\
\text { (74 DL) }\end{array}$ \\
\hline $\begin{array}{l}\text { Entre densité maximale et } \\
\text { densité minimale } . . . . . . .\end{array}$ & $-0,02$ & $-0,11$ & $0,43^{*}$ & $0.22^{\bullet}$ \\
\hline $\begin{array}{l}\text { Entre densité maximale et } \\
\text { largeur de cernes } . . . . . .\end{array}$ & 0,09 & $0,40^{*}$ & $-0,54^{* *}$ & $-0,36^{\circ 4}$ \\
\hline $\begin{array}{l}\text { Entre densité minimale et } \\
\quad \text { largeur de cernes } . . . . . .\end{array}$ & $-0,87^{* *}$ & $-0,72^{* *}$ & $-0,64^{* *}$ & $-0,74^{* *}$ \\
\hline
\end{tabular}

Si certaines corrélations très étroites apparaissent (celles de signe négatif qui existent entre densités minimales et largeurs de cernes en particulier méritent d'être notées), elles sont, somme toute, assez rares, compte tenu du fait que l'on compare des caractéristiques moyennes relevées à l'intérieur des mêmes cernes ; on n'a manifestement pas affaire à des liaisons fonctionnelles, et il y a, par suite, peu de chance pour que les indications fournies par les diverses variables se superposent exactement.

Pour le vérifier, on a repris la liste des 32 années sur lesquelles a porté l'expérience, et l'on a déterminé, d'une part, celles qui sont caractéristiques du seul point de vue de la dendrochronologie, c'est-à-dire d'après les largeurs de cernes, et d'autre part, celles qui possèdent au moins un critère caractéristique, que celui-ci soit de nature dendrochronologique ou xylochronologique ; les résultats sont consignés sur le tableau 10.

On voit que largeurs de cernes, densités maximales et densités minimales ne caractérisent absolument pas les mêmes années, et que les renseignements fournis par les unes et les autres se complètent au contraire utilement pour aider à la datation des accroissements.

L'apport de la xylochronologie permet, en gros, de doubler le nombre des années caractéristiques pour les parcelles 2 et 3 , de le tripler pour la parcelle 1, et pratiquement de le sextupler pour l'ensemble des arbres de l'expérience.

Le cas de la parcelle 3, c'est-à-dire du peuplement normal, est digne d'être sou- 
TABLEAU 10

Années présentant au moins une donnée caractéristique

\begin{tabular}{|c|c|c|c|c|c|c|c|c|}
\hline & \multicolumn{4}{|c|}{ DENDROCHRONOLOGIE } & \multicolumn{4}{|c|}{$\begin{array}{l}\text { DENDROCHRONOLOGIE } \\
+ \text { XYLOCHRONOLOGIE }\end{array}$} \\
\hline & Plle I & Plle 2 & Plle 3 & Total & Plle 1 & Plle 2 & Plle 3 & Total \\
\hline $1936 \ldots \ldots \ldots \ldots$ & \multirow{6}{*}{$x$} & \multirow{7}{*}{$\begin{array}{l}x \\
x\end{array}$} & \multirow[t]{3}{*}{$x$} & & $x$ & $x$ & $\times$ & \multirow{3}{*}{$\begin{array}{l}x \\
x\end{array}$} \\
\hline $1937 \ldots \ldots \ldots \ldots$ & & & & & $x$ & $x$ & \multirow[t]{2}{*}{$x$} & \\
\hline $1938 \ldots \ldots \ldots \ldots$ & & & & & $x$ & $x$ & & \\
\hline $1939 \ldots \ldots \ldots \ldots$ & & & \multirow[t]{2}{*}{$x$} & & $x$ & $x$ & $\mathrm{x}$ & \multirow[t]{2}{*}{$x$} \\
\hline $1940 \ldots \ldots \ldots \ldots$ & & & & & $x$ & & & \\
\hline $1941 \ldots \ldots \ldots \ldots$ & & & \multirow[t]{2}{*}{$x$} & & $x$ & $\times$ & $x$ & \multirow[t]{2}{*}{$x$} \\
\hline $1942 \ldots \ldots \ldots \ldots$ & $\times$ & & & & $x$ & & $x$ & \\
\hline $1943 \ldots \ldots \ldots \ldots$ & \multirow[t]{2}{*}{$x$} & $x$ & $x$ & $x$ & $\times$ & $x$ & $x$ & $x$ \\
\hline $1944 \ldots \ldots \ldots \ldots$ & & $x$ & $x$ & $x$ & $\times$ & $x$ & $x$ & $x$ \\
\hline $1945 \ldots \ldots \ldots \ldots$ & $x$ & $x$ & \multirow[t]{2}{*}{$x$} & & $x$ & $x$ & $x$ & \multirow[t]{2}{*}{$x$} \\
\hline $1946 \ldots \ldots \ldots \ldots$ & \multirow[t]{14}{*}{$x$} & \multirow[t]{5}{*}{$x$} & & & $x$ & $x$ & $x$ & \\
\hline $1947 \ldots \ldots \ldots \ldots$ & & & \multirow{3}{*}{$\begin{array}{l}x \\
x\end{array}$} & & $\mathrm{x}$ & $x$ & $x$ & \multirow{2}{*}{$\begin{array}{l}x \\
x\end{array}$} \\
\hline $1948 \ldots \ldots \ldots \ldots$ & & & & & $x$ & $x$ & $x$ & \\
\hline $1949 \ldots \ldots \ldots \ldots$ & & & & & $x$ & $x$ & $x$ & \multirow[t]{2}{*}{$\times$} \\
\hline $1950 \ldots \ldots \ldots \ldots$ & & & \multirow[t]{3}{*}{$x$} & & & & $x$ & \\
\hline $1951 \ldots \ldots \ldots \ldots$ & & \multirow[t]{3}{*}{$x$} & & & & $x$ & $x$ & $x$ \\
\hline $1952 \ldots \ldots \ldots \ldots$ & & & & & $x$ & $x$ & $x$ & $\mathrm{x}$ \\
\hline $1953 \ldots \ldots \ldots \ldots$ & & & \multirow[t]{3}{*}{$x$} & & & & $x$ & $x$ \\
\hline $1954 \ldots \ldots \ldots \ldots$ & & \multirow[t]{2}{*}{$\times$} & & & $x$ & $x$ & $\times$ & $x$ \\
\hline $1955 \ldots \ldots \ldots \ldots$ & & & & & & & $x$ & $\mathrm{x}$ \\
\hline $1956 \ldots \ldots \ldots \ldots$ & & \multirow[t]{3}{*}{$x$} & \multirow{6}{*}{$\mathrm{x}$} & & $x$ & $x$ & $x$ & \multirow{3}{*}{$x$} \\
\hline $1957 \ldots \ldots \ldots \ldots$ & & & & & & $x$ & $x$ & \\
\hline $1958 \ldots \ldots \ldots \ldots$ & & & & & & $x$ & $x$ & \\
\hline $1959 \ldots \ldots \ldots \ldots$ & & $\times$ & & & $x$ & $x$ & $x$ & $x$ \\
\hline $1960 \ldots \ldots \ldots \ldots$ & \multirow[t]{2}{*}{$x$} & & & & $x$ & $\times$ & $x$ & $x$ \\
\hline $1961 \ldots \ldots \ldots \ldots$ & & & & & $x$ & $x$ & $x$ & $x$ \\
\hline $1962 \ldots \ldots \ldots \ldots$ & $x$ & $\times$ & & $x$ & $x$ & $x$ & $x$ & $x$ \\
\hline $1963 \ldots \ldots \ldots \ldots$ & & & & & & $\times$ & $x$ & $x$ \\
\hline $1964 \ldots \ldots \ldots \ldots$ & $x$ & $x$ & & $x$ & $x$ & $x$ & $x$ & $x$ \\
\hline $1965 \ldots \ldots \ldots \ldots$ & & & & & $x$ & & $x$ & $\times$ \\
\hline $1966 \ldots \ldots \ldots \ldots$ & & & $\mathrm{x}$ & & $\times$ & & $x$ & \\
\hline $1967 \ldots \ldots \ldots \ldots$ & $x$ & $x$ & & & $x$ & $x$ & & \\
\hline & 9 & 13 & 12 & 4 & 25 & 25 & 29 & 23 \\
\hline
\end{tabular}

ligné, puisque, en utilisant à la fois les critères de croissance de l'arbre et ceux de la densité du bois, on arrive à avoir 29 années caractéristiques sur 32 .

\section{IV. - APPLICATIONS PRATIQUES AUX PROBLẺMES DE DATATION}

\section{1. - Profils XYLOCHRONOLOGiQues SYNTHÉTIQUES}

Dans cette étude, nous nous sommes volontairement abstenus de faire appel à un appareillage complexe ou de nous livrer à des calculs qui eussent rendu obliga- 
toire le recours à de puissants ordinateurs, ceci précisément dans le but de montrer que les problèmes de datation pouvaient être résolus de façon simple, en utilisant les ressources de la xylochronologie.

Pour la même raison, toutes les déterminations dont il est rendu compte ici ont été faites sur des données brutes, sans transformation logarithmique et sans artifice tel que l'affectation à une année de la moyenne arithmétique entre la donnée la concernant en propre, celle de l'année précédente et celle de l'année suivante, ou encore d'une moyenne pondérée par l'attribution d'un double coefficient à l'année en cause.

Malgré cette simplification considérable de la procédure, l'étude ci-dessus a montré que l'ensemble des trois données élémentaires : largeurs des cernes, densités maximales et densités minimales, permettait en général des datations très faciles, en dépit de la présence de cernes extrêmement fins, ou même de l'absence de tout accroissement pour une année donnée.

Un des moyens les plus commodes pour y parvenir consiste à réaliser tout d'abord des profils moyens synthétiques à partir des échantillons dont la datation ne pose pas de problèmes, et à leur comparer les courbes individuelles des arbres à cernes douteux.

On a donné le nom de "profils xylochronologiques synthétiques» à des graphiques où l'on a porté, en abscisse, les largeurs de cernes, et en ordonnée, les densités minimales et les densités maximales, et où l'on a rejoint par un segment de droite les points figuratifs des minima et des maxima de densité annuels; en somme, on conserve les valeurs extrêmes de densité des courbes densitométriques, mais on supprime toutes les indications intermédiaires : pourcentages de bois de densité supérieure ou égale à un seuil donné, pente de la courbe entre bois initial et bois final, ou autres critères de forme.

Bien que l'on néglige ainsi des renseignements dont on a vu qu'ils facilitaient grandement l'identification des accroissements, de tels graphiques sont en général suffisants pour résoudre les problèmes de datation, grâce à la possibilité qu'ils offrent de représenter de façon schématique les variations annuelles simultanées de deux caractéristiques indépendantes, alors que les courbes dendrochronologiques classiques ne rendent compte que des modifications d'une seule d'entre elles : la largeur de cernes.

\section{2. - SYNCHRONISATION PROPREMENT DITE}

Sur la figure 5 sont regroupés, en traits pleins, les profils synthétiques moyens des trois parcelles concernées par cette expérience, ainsi que, en traits interrompus, ceux des deux arbres pour lesquels des problèmes de datation se sont posés (à noter que, pour la parcelle 3 et pour l'arbre 58 , l'échelle des largeurs de cernes est deux fois plus faible que pour les autres profils). 
PROFILS XYLOCHRONOLOGIQUES SYNTHETIQUES
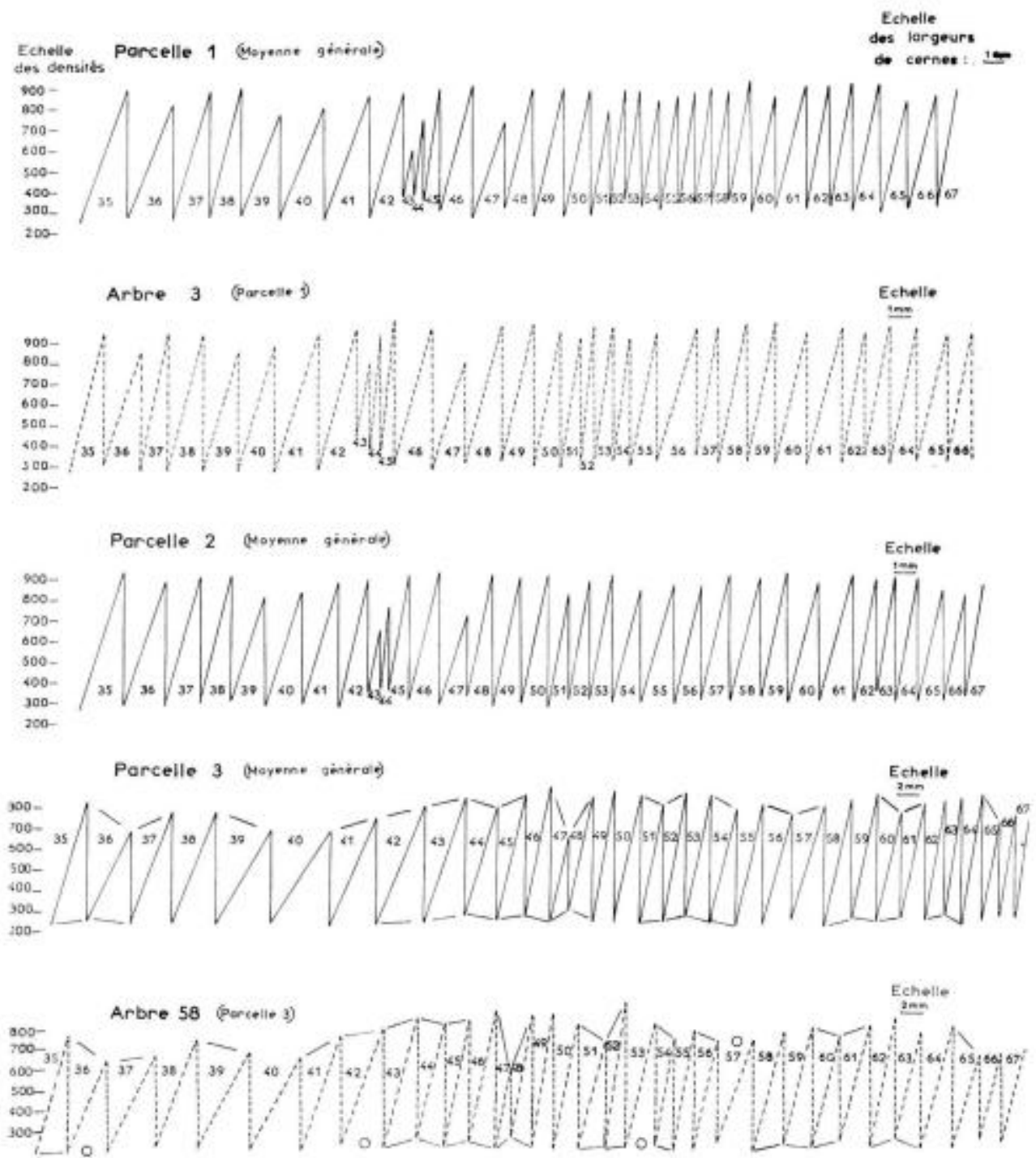

Fig. 5.

\subsection{1. - Cas de l'arbre $n^{0} 3$}

Il n'est mentionné ici que parce que l'expéditeur suisse des échantillons avait signalé, dans sa lettre d'envoi, qu'il manquait à la carotte de sondage de cet arbre, trois cernes du côté de l'écorce. 
La seule comparaison de son profil synthétique avec le profil moyen de la parcelle montre qu'il n'en manquait en fait qu'un seul, et la chose est tellement évidente que tout autre commentaire serait superflu.

On doit d'ailleurs reconnaître objectivement que, dans le cas particulier, la seule séquence des largeurs de cernes 1943 - 1944 - 1945 aurait probablement suffi à rétablir le nombre de cernes exact.

\subsection{2. - Cas de Tarbre $n^{\circ} 58$}

$\mathrm{Si}$ on se reporte à la courbe densitométrique de cet échantillon (fig. 3), on voit que l'on a apparemment 8 cernes après celui qui a finalement été attribué à l'année 1960, alors que la récolte des échantillons avait eu lieu avant la pousse 1968 ; or, la densité maximale du cerne 1947 est tellement caractéristique dans tous les arbres de l'expérience qu'il y avait peu de chance pour que la synchronisation de la période antérieure à 1960 soit erronée.

On a done à nouveau comparé le profil synthétique de l'arbre litigieux au profil moyen de la parcelle 3 , mais, la solution n'étant pas aussi évidente que dans le cas précédent, on a reporté sur le profil de base les années caractéristiques au point de vue xylochronologique, en indiquant pour chacune le sens de variations (augmentation ou diminution par rapport à l'année précédente).

Sur le profil de l'arbre 58 portant la synchronisation que l'on supposait exacte, on a souligné par les mêmes traits ascendants et descendants les accroissements où le sens de variations était le même que sur le profil moyen, et par 0 ceux oủ il y avait, au contraire, divergence ; on a obtenu ainsi une coïncidence pour 17 cernes sur 18 au point de vue densité maximale, et pour 15 sur 18 au point de vue densité minimale ; on était par suite pratiquement assuré que la synchronisation proposée était correcte, puisque bien entendu aucun changement d'origine de la datation ne pouvait donner une telle similitude de résultats.

En s'intéressant de plus près aux derniers accroissements, on a constaté que la datation des cernes 1962 et 1963 d'une part, et 1965 d'autre part, était, selon toute probalité, exacte puisqu'on y retrouve les sens de variations caractéristiques de l'ensemble de la parceile ; on s'est finalement aperçu que l'année 1964 était, sur la courbe densitométrique, représentée par ce qui semblait être deux cernes accolés (et non pas par un double cerne classique beaucoup plus facilement repérable) ; un examen plus approfondi de la radiographie et de l'échantillon lui-même a confirmé que celuici recelait une fente interne extrêmement mince dans cet accroissement, fente qui s'était traduite par un pic aberrant vers les faibles densités à l'intérieur du bois final, donnant ainsi à l'exploration densitométrique l'apparence d'un cerne supplémentaire.

\section{CONCLUSION}

L'expérience dont il est rendu compte ici montre que les densités maximales et les densités minimales annuelles, tout en étant sans aucun doute sous la dépendance 
étroite des facteurs climatiques, sont assez peu sensibles, au contraire, aux autres sources de variations : environnement ou vieillissement de l'assise génératrice ; comme telles, elles fournissent un indicatif de choix pour la datation des accroissements.

La xylochronologie, qui est basée sur de tels critères, ne doit pas, semble-t-il, concurrencer la dendrochronologie classique, mais seulement la compléter, la première apportant des données nouvelles au moment où la seconde paraît s'orienter vers I'utilisation de méthodes mathématiques plus élaborées.

Ensemble, elles doivent permettre des synchronisations à la fois plus faciles, plus rapides et plus sûres ; ensemble aussi, elles sont susceptibles de recevoir des applications pratiques plus nombreuses, en particulier dans le domaine de la connaissance du climat des siècles passés.

Recu pour publication en janvier 1969

\section{SUMMARY}

THE XYLOCHRONOLOGY, HELPFUL COMPLEMENT TO THE DENDROCHRONOLOGY

The word " xylochronology $"$ is applied to datation studies based on wood characteristics (particularly the maximum and minimum annual densities) instead of the alone ringwidth which is used in « dendrochronology".

The material for this experience was made of samples collected from a swiss forest strongly damaged by industrial smokes (Plot 1), from an intermediate stand (Plot 2) and from a control stand (Plot 3) which was younger than the others.

On the figures 1,2 and 3 , we can see that the shapes of the densitometric records often are very typical for a given year (particularly for the years 1939 and 1940, 1943, 1947 and 1960).

The percentages of coincidence are lower for the ringwidths than for the maximum and minimum densities (appendix 1 to 9 ). The number of percentages above $75 \%$ is roughly 4 times higher for density criteria than for the widths of the annual increments (table 2).

The characteristic years also are more numerous for the maximum and minimum densities (table 3 to 5): on table 6 are indicated the years for which the data are higher $(\mathrm{M})$ or lower $(\mathrm{m})$ than these of the preceding year for $80 \%$ of the trees. For all the plots, the number of such years is 3 times higher (maximum densities) or 4 times higher (minimum densities) for densitometric criteria than for ringwidths.

On figure 4 are shown the variations of mean values per plot for the different data. The dispersion between the stands 1 and 2 and the stand 3 is lower for the density characteristics and confirmation is given by the coefficients of variation (table 7). Elsewhere the coefficients of correlation between the mean values per plot are lower for the ringwidths (table 8 ) and all these observations prove that the maximum and minimum annual densities strongly depend upon climatic factors but are less sensititive than ringwidths to other sources of variation ; industrial damages or ageing of the cambium.

The table 10 shows that the width and the density criteria don't characterize the same years and so together they are able to make easier the datation of the annual increments.

At last the construction of synthetic xylochronological profiles, of which the abcissa are the ringwidths and the ordinates the maximun and minimum density values, strongly helps to the solution of the synchronisation problems.

\section{ZUSAMMENFASSUNG}

DIE XYLOCHRONOLOGIE, EINE HILEREICHE ERGÄNZUNG DER DENDROCHRONOLOGIE

Unter Xylochronologie wird die Datierung der Jahrringe an Hand von Holzeigenschaften - insbesondere die maximale und minimale Dichte - verstanden, während die Dendrochronologie nur die Jahrringbreite berücksichtigt. 
Das Untersuchungsmaterial (Bohrspăne) stammt aux einem Waldgebiet der Schweiz, Die Parzelle 1 war sehr stark rauchgeschädigt, die Parzelle 2 wies mittlere Rauchschāden auf, während die Parzelle 3, welche ein geringeres Alter aufweist und nicht rauchgeschädigt ist, als Vergleichsfläche herangezogen wurde.

Die Abbildungen 1,2 und 3 stellen Dichteregistrierungen von Bohrspänen dar, welche oftmals eine für ein gegebenes Jahr typische Form aufweisen, insbesondere für die Jahre 1939, 1940, 1943, 1947 und 1960.

Die prozentuale Koinzidenz ist bei der Jahrringbreite geringer als bei der maximalen und minimalen Dichte (Anhang 1 - 9). Bei den Dichtekriterien sind Koinzidenzprozente von über $75 \%$ viermal häufiger als bei den Jahrringbreiten (Tabelle 2). Charakteristische Jahre bezüglich der maximalen und minimalen Dichte sind gleichfalls zahlreicher als bei den Jahrringbreiten (Tabellen 3 - 5).

In Tabelle 6 sind jene Jahre wiedergegeben, bei welchen die verschiedenen Werte für $80 \%$ der Bäume höher $(\mathrm{M})$ oder geringer $(\mathrm{m})$ als im Vorjahr waren. In allen drei Parzellen ist die Anzahl dieser Jahre dreimal (maximale Dichte) oder viermal grösser (minimale Dichte) bei den Dichtekriterien als bei den Jahrringbreiten.

Die Abbildung 4 gibt die Variationen der Parzellenmittelwerte für die verschiedenen gemessenen Grössen wieder. Die Dispersion zwischen den Parzellen 1 und 2 und der Parzelle 3 ist bei den Dichtecharakteristiken geringer und wird durch die Variationskoeffizienten bestätigt (Tabelle 7). Desweiteren sind die Korrelationskoeffizienten zwischen den Parzellenmittelwerten bei den Jahrringbreiten geringer (Tabelle 8 ) und alle diese Ergebnisse zeigen, das die Maximal- und Minimaldichte des Jahrringes sehr stark von klimatischen Faktoren abhängt, jedoch von anderen Faktoren, wie z. B. Industricabgase oder Alterung des Kambiums, nur wenig beeinflusst werden.

Die Tabelle 10 zeigt, dass Jahrringbreiten und Dichtekriterien nicht für dieselben Jahre kennzeichnend sind, sodass bei Verwendung der beiden Kriterien eine wesentlich bessere Datierung der Jahrringe möglich wird.

Abschliessend sei noch erwăhnt, dass die Aufstellung von synthetischen xylochronologischen Profilen mit den Jahrringbreiten als Abszissen und der Maximal- und Minimaldichte als Ordinate die Lösung von Synchronisationsproblemen wesentlich erleichtert.

\section{RÉFÉRENCES BIBLIOGRAPHIQUES}

ANTEVs E., 1925. The big trees as a climatic measure Quaternary Climates. Carnegie. Inst. Wash. Publ. (352), 115-153.

BANNister B,, 1963, Dendrochronology, p. 162-176. In D. Brothwell and E. Higgs (ed.) Science and Archaeology. Basic Book Inc., New York.

Corona E., 1959. Indagini di cronologia anulare. Studi Trentini di Scienze Naturali. (1), 49-55.

Douglass A.E., 1919. Climatic cycles and tree-growth. Carnegie Inst. Wash. Publ., 289, (2), 1-166.

Douglass A.E., 1920. Evidence of climatic effects in the annual ring of trees. Ecology, 1, 24-32.

Druce A., 1966. Tree-ring dating of recent volcanic ash and lapilli, mt egmont. New Zeal, J. Bot., 4. (1).

Eluort G.K., BRоoк S.E.G., 1967, A microphotometric technique for growth-ring analysis. J. Inst. Wood Sci., (18).

FloRence J., 1962. Exemple d'étude dendrologique en relation avec la climatologie du Capcir et de la Cerdagne. Thèse de $3^{\circ}$ cycle de Biogéographie. Faculté des Sciences de Toulouse.

Fritrs H.C., 1963, Computer programs for tree-ring research. Tree-ring Bull., 25, (3-4), 2-7.

FrITIS H.C., 1965. Tree-ring evidence for climatic changes in Western North America. Monthly Weather Rev., 93, (7), 421-443.

Fritts H.C., Sмrth D.G., Cardis J.W., and Budelsky C.A., 1965. Tree-ring characteristics along a vegatation gradient in Northerm Arizona. Ecology, 46, (4).

Fritrs H.C., 1962. An approach to dendroclimatology : screening by means of multiple regression techniques.

GrefNe H.V., Worral. J., 1964. Wood quality studies. I - A scanning microphotometer for automatically measuring and recording certain wood characteristics. Tappi, 47, 7, 419-427. 
Huber Br., 1943. Uber die Sicherheit jahrringchronologischer Datierung. Holz als Roth- und Werkstoff, 6, (10-12), 263-268.

Munaut A.V., 1966. Recherches dendrochronologiques sur Pinus silvestris. I - Etude sur 45 Pins sylvestres récents originaires de Belgique. Agriculture, 14, (2), $2^{*}$ série.

Philurs E.W.J., 1960. The beta ray method of determining the density of wood and the proportion of summer wood. J. Inst. Wood Sci., (5-8).

Polge H., 1966. Etablissement des courbes de variation de la densité du bois par exploration densitométrique de radiographie d'échantillons prélevés à la tariére sur des arbres vivants. Applications dans les domaines technologique et physiologique. Thèse Docteur és Sciences, Faculté des Sciences de Nancy Ann, Sci. forest., 23, (1).

POLLANsCHÜTZ 1962. Rauchschadenfeststellung unter besonder Berücksichtigung von Bohrkeranalysn. Forstliche Bundesversuchanstalt Mariabrunn, Informationsdienst, April.

SERRE F., 1966. Recherches préliminaires sur les 23 relations entre les variations des accroissements annuels du Pin d'Alep et celle des facteurs climatiques. Oecol. Plant., (1), 117-135.

Schulman E., 1955. Dendroclimatic changes in semi-arid Ametrica. 142 p. Tucson Univ. of Arizona Press.

VINs, 1962. Použiti letokruhovych analys k prukazu kourovych shod-Rocvik 8 (XXXV), 4, 23-280. 


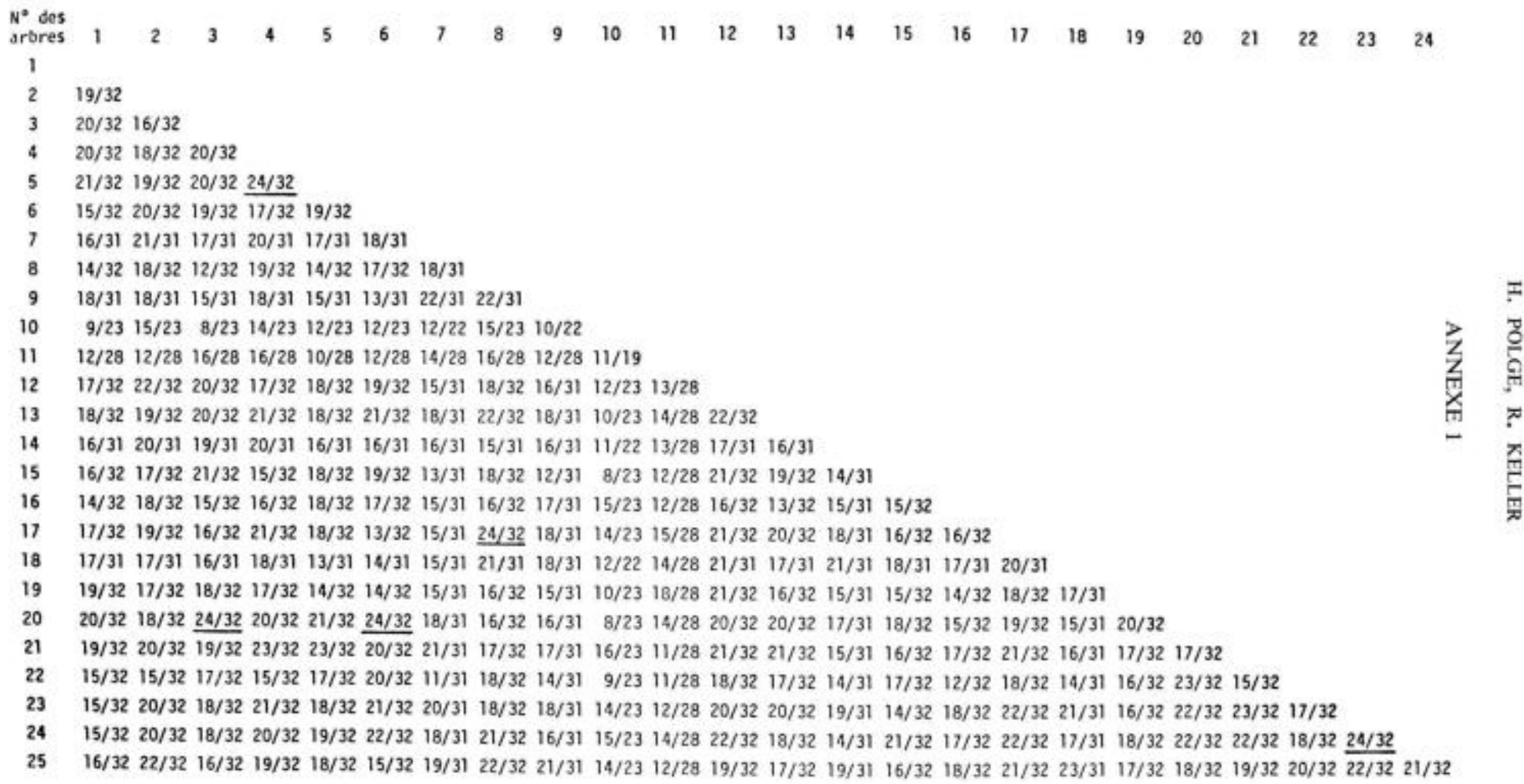




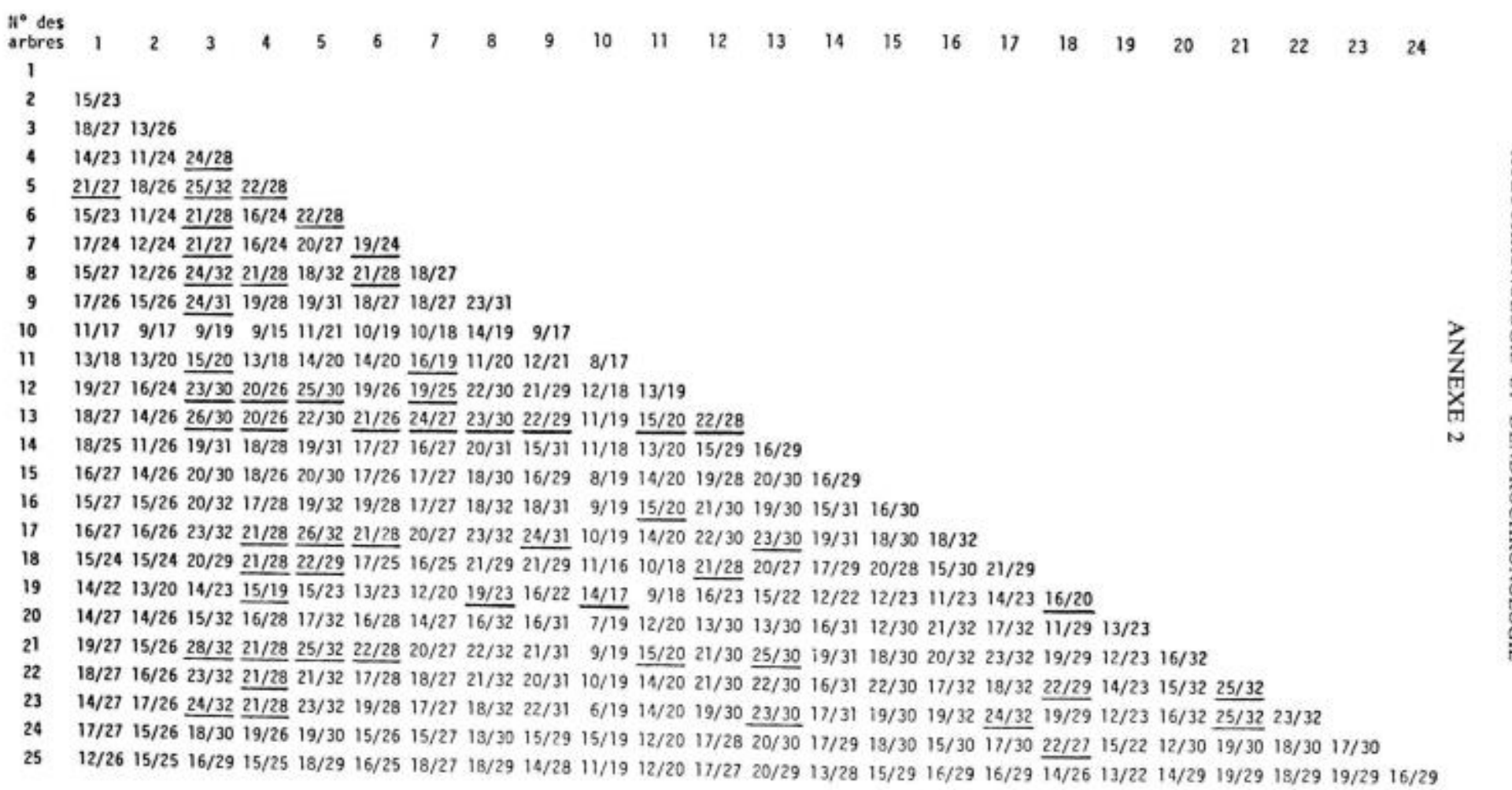




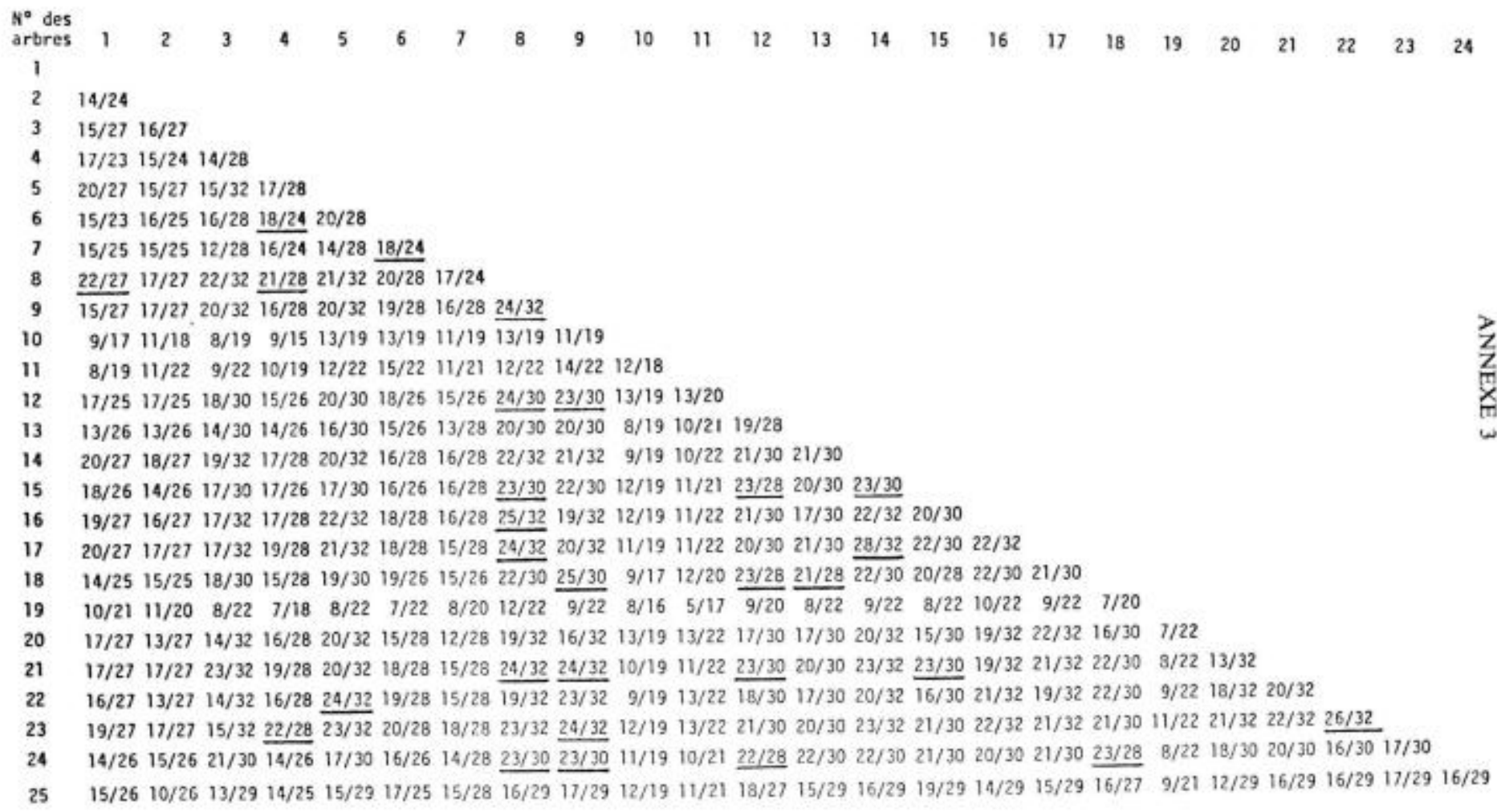




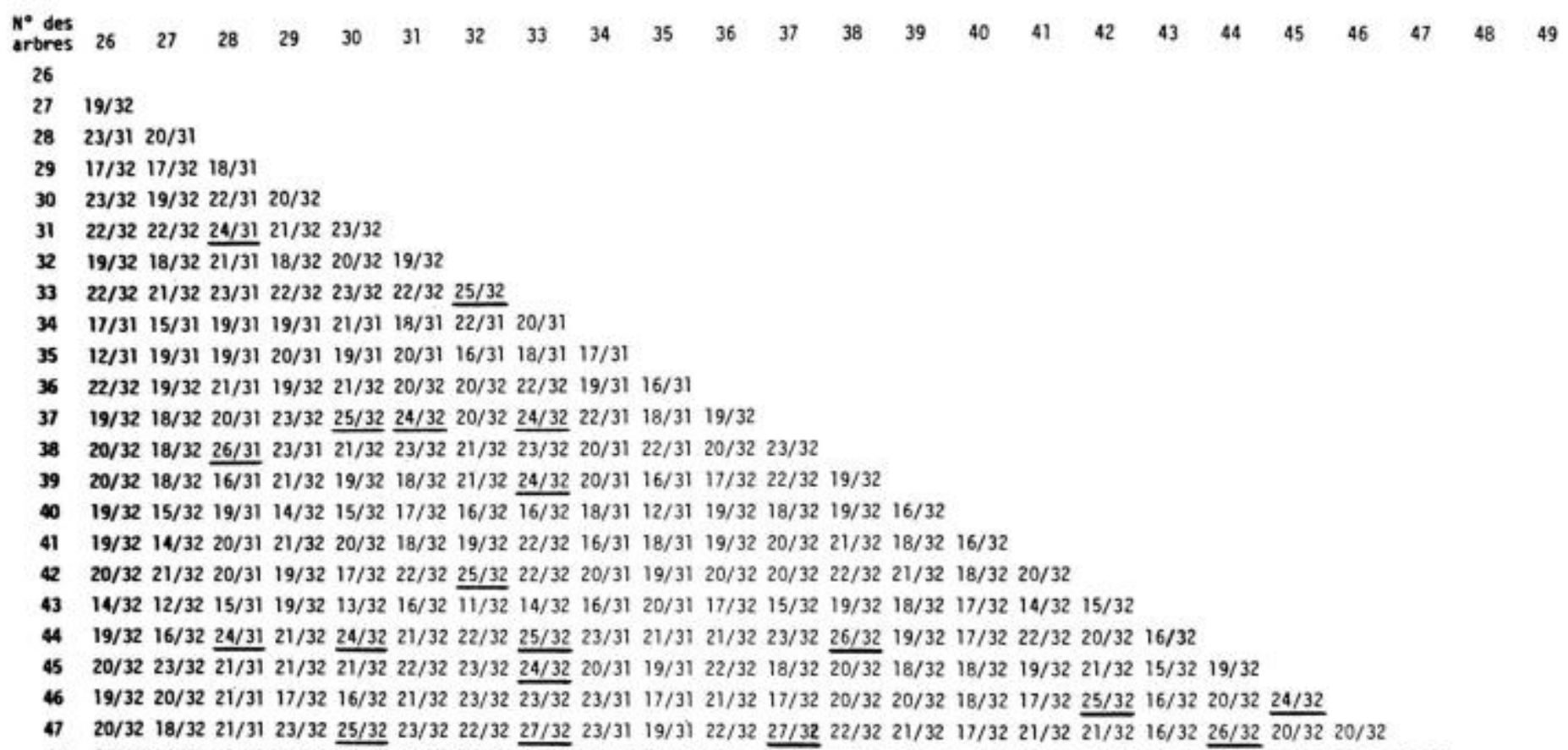




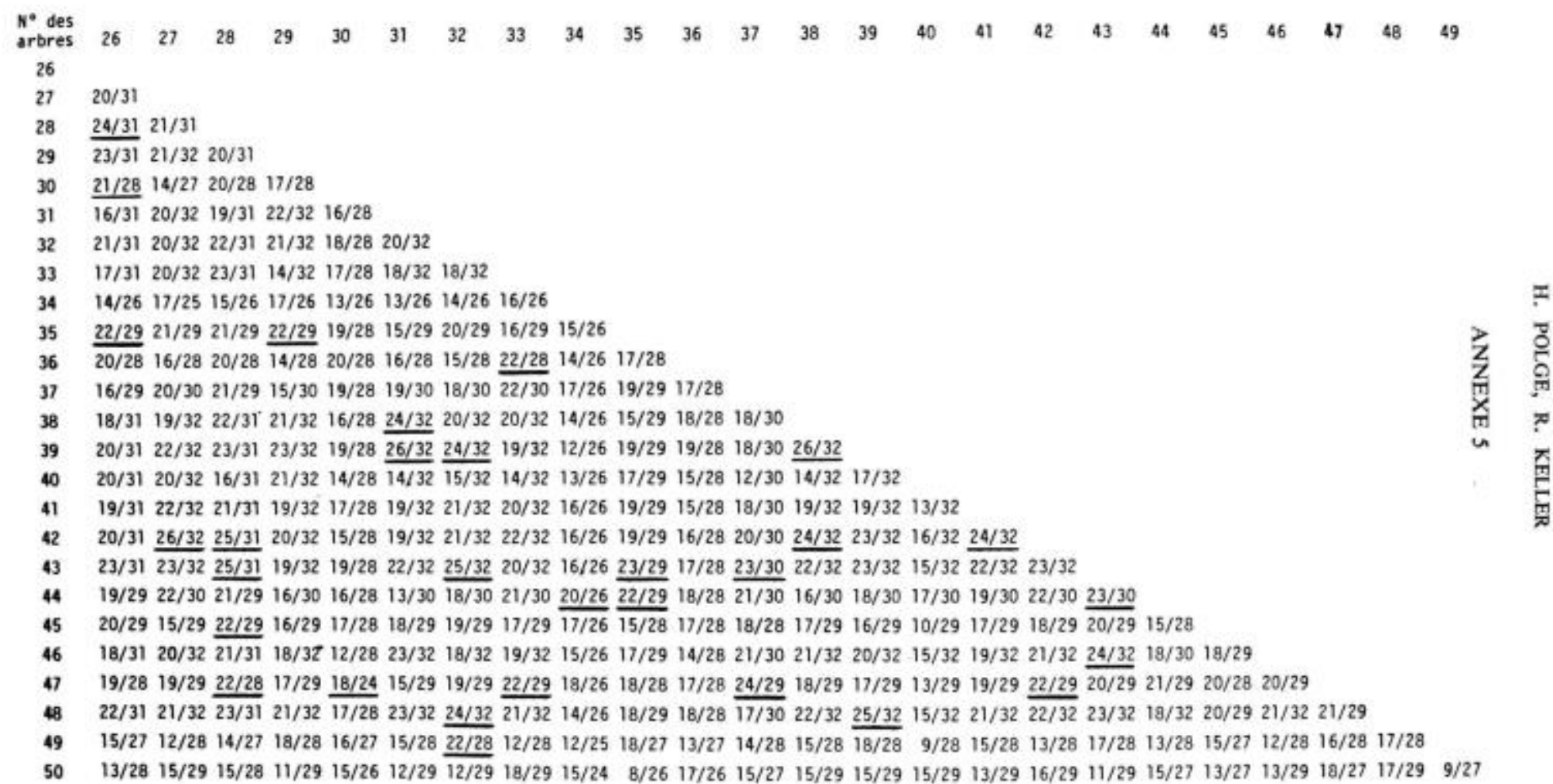




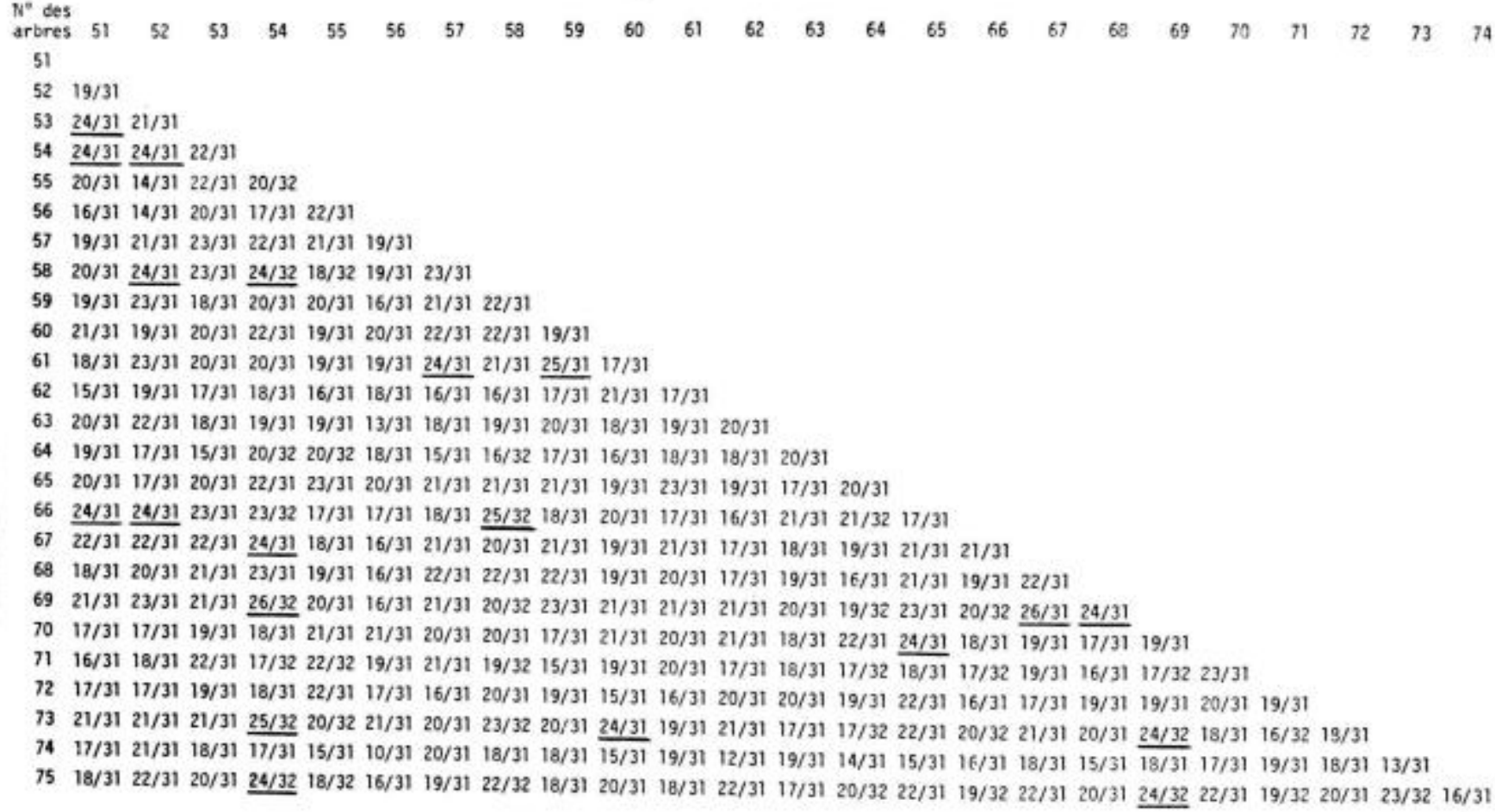

$52 \quad 19 / 31$

$53 \quad 24 / 31 \quad 21 / 31$

$54 \quad 24 / 3124 / 31 \quad 22 / 31$

$55 \frac{20 / 31}{14 / 31} 22 / 3120 / 32$

$56 \quad 16 / 31 \quad 14 / 31 \quad 20 / 31 \quad 17 / 31 \quad 22 / 31$

57 19/31 21/31 23/31 22/31 21/31 19/31

58 20/31 $24 / 31 \quad 23 / 31 \quad 24 / 32 \quad 18 / 32 \quad 19 / 31 \quad 23 / 31$

$5919 / 31 \quad 23 / 31 \quad 18 / 31 \quad 20 / 31 \quad 20 / 31 \quad 16 / 31 \quad 21 / 31 \quad 22 / 31$

$\begin{array}{llllllllll}60 & 21 / 31 & 19 / 31 & 20 / 31 & 22 / 31 & 19 / 31 & 20 / 31 & 22 / 31 & 22 / 31 & 19 / 31\end{array}$

$\begin{array}{lllllllllll}61 & 18 / 31 & 23 / 31 & 20 / 31 & 20 / 31 & 19 / 31 & 19 / 31 & 24 / 31 & 21 / 31 & 25 / 31 & 17 / 31\end{array}$

62 15/31 19/31 $17 / 31 \quad 18 / 31 \quad 16 / 31 \quad 18 / 31 \quad 16 / 31 \quad 16 / 31 \quad 17 / 31 \quad 21 / 31 \quad 17 / 31$

$63 \quad 20 / 31 \quad 22 / 31$ 18/31 19/31 19/31 13/31 18/31 19/31 20/31 18/31 19/31 20/31

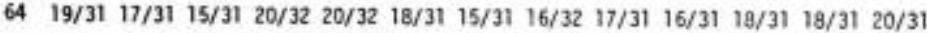

65 20/31 17/31 20/31 22/31 23/31 20/31 $21 / 31 \quad 21 / 31 \quad 21 / 31 \quad 19 / 31 \quad 23 / 31 \quad 19 / 31 \quad 17 / 31 \quad 20 / 31$

$66 \quad \frac{24 / 31}{2} \frac{24 / 31}{23 / 31} 23 / 32 \quad 17 / 31 \quad 17 / 31 \quad 18 / 31 \quad 25 / 32 \quad 18 / 31 \quad 20 / 31 \quad 17 / 31 \quad 16 / 31 \quad 21 / 31 \quad 21 / 32 \quad 17 / 31$

$67 \quad 22 / 3122 / 31 \quad 22 / 31 \quad 24 / 31 \quad 18 / 31$ 16/31 21/31 $20 / 31 \quad 21 / 31 \quad 19 / 31 \quad 21 / 31 \quad 17 / 31 \quad 18 / 31 \quad 19 / 31 \quad 21 / 31 \quad 21 / 31$

68 18/31 20/31 21/31 23/31 19/31 16/31 22/31 22/31 22/31 $19 / 31$ 20/31 $17 / 31$ 19/31 $16 / 31 \quad 21 / 31$ 19/31 $22 / 31$

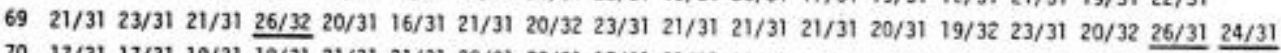

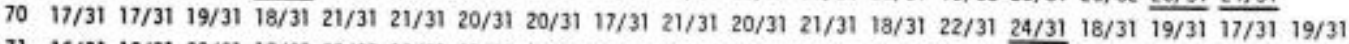

$\begin{array}{lllllllllllllllllllll}71 & 16 / 31 & 18 / 31 & 22 / 31 & 17 / 32 & 22 / 32 & 19 / 31 & 21 / 31 & 19 / 32 & 15 / 31 & 19 / 31 & 20 / 31 & 17 / 31 & 18 / 31 & 17 / 32 & 18 / 31 & 17 / 32 & 19 / 31 & 16 / 31 & 17 / 32 & 23 / 31\end{array}$

72 17/31 17/31 19/31 18/31 22/31 17/31 16/31 20/31 19/31 15/31 16/31 20/31 20/31 19/31 22/31 16/31 $17 / 31$ 19/31 19/31 20/31 19/31

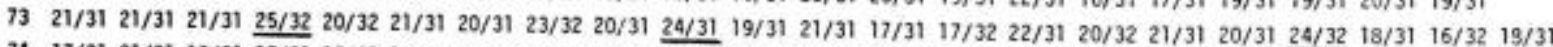

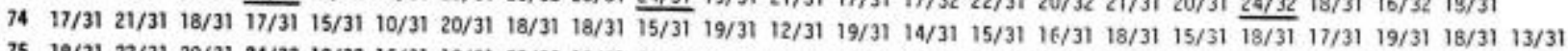

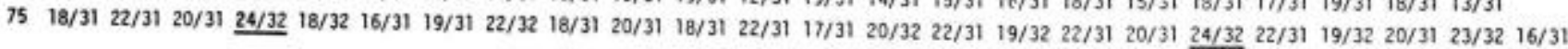


Pourcentages de coincidence entre tous les arbres pris deux à deux. Parcelle 3. Densités maximales

\begin{tabular}{|c|c|c|c|c|c|c|c|c|c|c|c|c|c|c|c|c|c|c|c|c|c|c|c|c|}
\hline $\begin{array}{l}N^{*} \text { des } \\
\text { arbres }\end{array}$ & 51 & 52 & 53 & 54 & 55 & 56 & 57 & 58 & 59 & 60 & 61 & 62 & 63 & 64 & 65 & 66 & 67 & 68 & 69 & 70 & 71 & 72 & 73 & 74 \\
\hline 51 & & & & & & & & & & & & & & & & & & & & & & & & \\
\hline 52 & $23 / 31$ & & & & & & & & & & & & & & & & & & & & & & & \\
\hline 53 & $16 / 31$ & $17 / 31$ & & & & & & & & & & & & & & & & & & & & & & \\
\hline 54 & $26 / 31$ & $24 / 31$ & $14 / 31$ & & & & & & & & & & & & & & & & & & & & & \\
\hline 55 & $21 / 31$ & $16 / 31$ & 26/31 & 18/32 & & & & & & & & & & & & & & & & & & & & \\
\hline 56 & $23 / 31$ & $20 / 31$ & $20 / 31$ & 19/31 & $21 / 31$ & & & & & & & & & & & & & & & & & & & \\
\hline 57 & $23 / 31$ & $20 / 31$ & $15 / 31 ?$ & $25 / 31$ & $18 / 31$ & $21 / 31$ & & & & & & & & & & & & & & & & & & \\
\hline 58 & $24 / 31$ & $25 / 31$ & $15 / 31 ?$ & $\underline{26 / 32}$ & $20 / 32$ & $21 / 31$ & $25 / 31$ & & & & & & & & & & & & & & & & & \\
\hline 59 & $19 / 31$ & $17 / 31$ & $15 / 31$ & $19 / 32$ & $16 / 32$ & $18 / 31$ & $20 / 312$ & $20 / 32$ & & & & & & & & & & & & & & & & \\
\hline 60 & $17 / 31$ & $21 / 31$ & $17 / 31$ & $20 / 31$ & $14 / 31$ & $18 / 31$ & $19 / 311$ & $19 / 31$ & $14 / 31$ & & & & & & & & & & & & & & & \\
\hline 61 & $20 / 31$ & $22 / 31$ & $18 / 31$ & $25 / 31$ & $21 / 31$ & $17 / 31$ & $21 / 312$ & $23 / 31$ & $15 / 31$ & $20 / 31$ & & & & & & & & & & & & & & 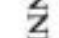 \\
\hline 62 & $25 / 31$ & $20 / 31$ & $22 / 31$ & $22 / 32=$ & $26 / 32$ & $25 / 31$ & $24 / 312$ & $24 / 32$ & $19 / 32$ & $18 / 31$ & 19/31 & & & & & & & & & & & & & गु \\
\hline 63 & $22 / 31$ & $21 / 31$ & $23 / 31$ & $20 / 31 ?$ & $24 / 31$ & $20 / 31$ & $19 / 312$ & $20 / 31$ & $21 / 31$ & $19 / 31$ & $22 / 31$ & $26 / 31$ & & & & & & & & & & & & ता \\
\hline 64 & $20 / 29$ & $16 / 29$ & $19 / 29$ & $18 / 30$ & $21 / 30$ & $21 / 29$ & $19 / 29$ & $19 / 30$ & $18 / 30$ & $16 / 29$ & $19 / 29$ & $\underline{23 / 30}$. & $23 / 30$ & & & & & & & & & & & $\infty$ \\
\hline 65 & 19/28 & $\underline{22 / 28}$ & $14 / 28$ & $21 / 28$ & $14 / 28$ & $18 / 28$ & $20 / 28$ & $21 / 28$ & $19 / 28$ & $19 / 28$ & $21 / 28$ & $19 / 28$ & $20 / 28$ & $19 / 28$ & & & & & & & & & & \\
\hline 66 & $\underline{25 / 31}$ & $23 / 31$ & $19 / 31$ & $25 / 32$ & $19 / 32$ & $22 / 31$ & $22 / 31$ & $23 / 32$ & $20 / 32$ & $20 / 31$ & $21 / 31$ & $26 / 32$ & 25/31 & $19 / 30$ & $21 / 28$ & & & & & & & & & \\
\hline 67 & $26 / 31$ & $\frac{24 / 31}{1}$ & $17 / 31$ & $27 / 31$ & $21 / 31$ & $19 / 31$ & $24 / 31$ & $25 / 31$ & $19 / 31$ & $18 / 31$ & $25 / 31$ & $23 / 31$ & $22 / 31$ & $18 / 30$ & $22 / 28$ & $24 / 31$ & & & & & & & & \\
\hline 68 & $23 / 31$ & $24 / 31$ & $14 / 31$ & $26 / 31$ & $18 / 31$ & $18 / 31$ & $22 / 31$ & $24 / 31$ & $16 / 31$ & $20 / 31$ & $26 / 31$ & $20 / 31$ & $20 / 31$ & $15 / 29$ & $20 / 28$ & $23 / 31$ & $25 / 32$ & & & & & & & \\
\hline 69 & $23 / 31$ & $23 / 31$ & $16 / 31 ?$ & $24 / 31$ & $19 / 31$ & $19 / 31$ & $19 / 31$ & $22 / 31$ & $17 / 31$ & $20 / 31$ & $23 / 31$ & $22 / 31$ & $24 / 31$ & $19 / 29$ & $20 / 28$ & $26 / 31$ & $22 / 31$ & $24 / 31$ & & & & & & \\
\hline 70 & $22 / 31$ & $24 / 31$ & $20 / 31$ & $25 / 31$ & $20 / 31$ & $19 / 31$ & $24 / 31$ & $23 / 31$ & $16 / 31$ & $20 / 31$ & $25 / 31$ & $23 / 31$ & $22 / 31$ & $16 / 29$ & $20 / 28$ & $22 / 31$ & $25 / 31$ & $22 / 31$ & $21 / 31$ & & & & & \\
\hline 71 & $19 / 31$ & $22 / 31$ & $13 / 31$ & $22 / 32$ & $16 / 32$ & $16 / 31$ & $22 / 31$ & $24 / 32$ & $17 / 32$ & $18 / 31$ & $23 / 31$ & $17 / 32$ & $18 / 31$ & $16 / 29$ & $20 / 28$ & $19 / 32$ & $20 / 31$ & $21 / 31$ & $18 / 31$ & $24 / 31$ & & & & \\
\hline 72 & $24 / 31$ & $23 / 31$ & $16 / 31$ & $24 / 31$ & $21 / 31$ & $21 / 31$ & $24 / 31$ & $28 / 31$ & $19 / 31$ & $17 / 31$ & $22 / 31$ & $25 / 31$ & $22 / 31$ & $20 / 29$ & $\underline{21 / 28}$ & $24 / 31$ & $24 / 31$ & $23 / 31$ & $24 / 31$ & $22 / 31$ & $23 / 31$ & & & \\
\hline 73 & $27 / 31$ & $23 / 31$ & $14 / 31$ & $29 / 32$ & $21 / 32$ & $20 / 31$ & $27 / 31$ & $29 / 32$ & $20 / 32$ & $19 / 31$ & $24 / 31$. & $25 / 32$ & $21 / 31$ & $21 / 30$ & $\underline{22 / 28}$ & $24 / 32$ & $28 / 31$ & $25 / 31$ & $23 / 31$ & $23 / 31$ & $23 / 31$ & $27 / 31$ & & \\
\hline 74 & $22 / 31$ & $21 / 31$ & $13 / 31$ & $25 / 31$ & $18 / 31$ & $18 / 31$ & $25 / 31$ & $\underline{26 / 31}$ & $17 / 31$ & $19 / 31$ & $24 / 31$ & $20 / 31$ & $19 / 31$ & $17 / 29$ & $19 / 28$ & $20 / 31$ & $23 / 31$ & $22 / 31$ & $20 / 31$ & $24 / 32$ & $27 / 31$ & $25 / 31$ & $25 / 31$ & \\
\hline 75 & $23 / 31$ & $23 / 31$ & $17 / 31$ & $26 / 32$ & $21 / 32$ & $20 / 31$ & $23 / 31$ & $\underline{24 / 32}$ & $20 / 32$ & $23 / 31$ & $24 / 31$ & $22 / 32$ & $21 / 31$ & $17 / 30$ & $22 / 28$ & $25 / 32$ & $23 / 31$ & $24 / 31$. & $24 / 31$ & $26 / 31$ & $22 / 32$ & $23 / 31$ & $\underline{25 / 32}$ & $23 / 31$ \\
\hline
\end{tabular}




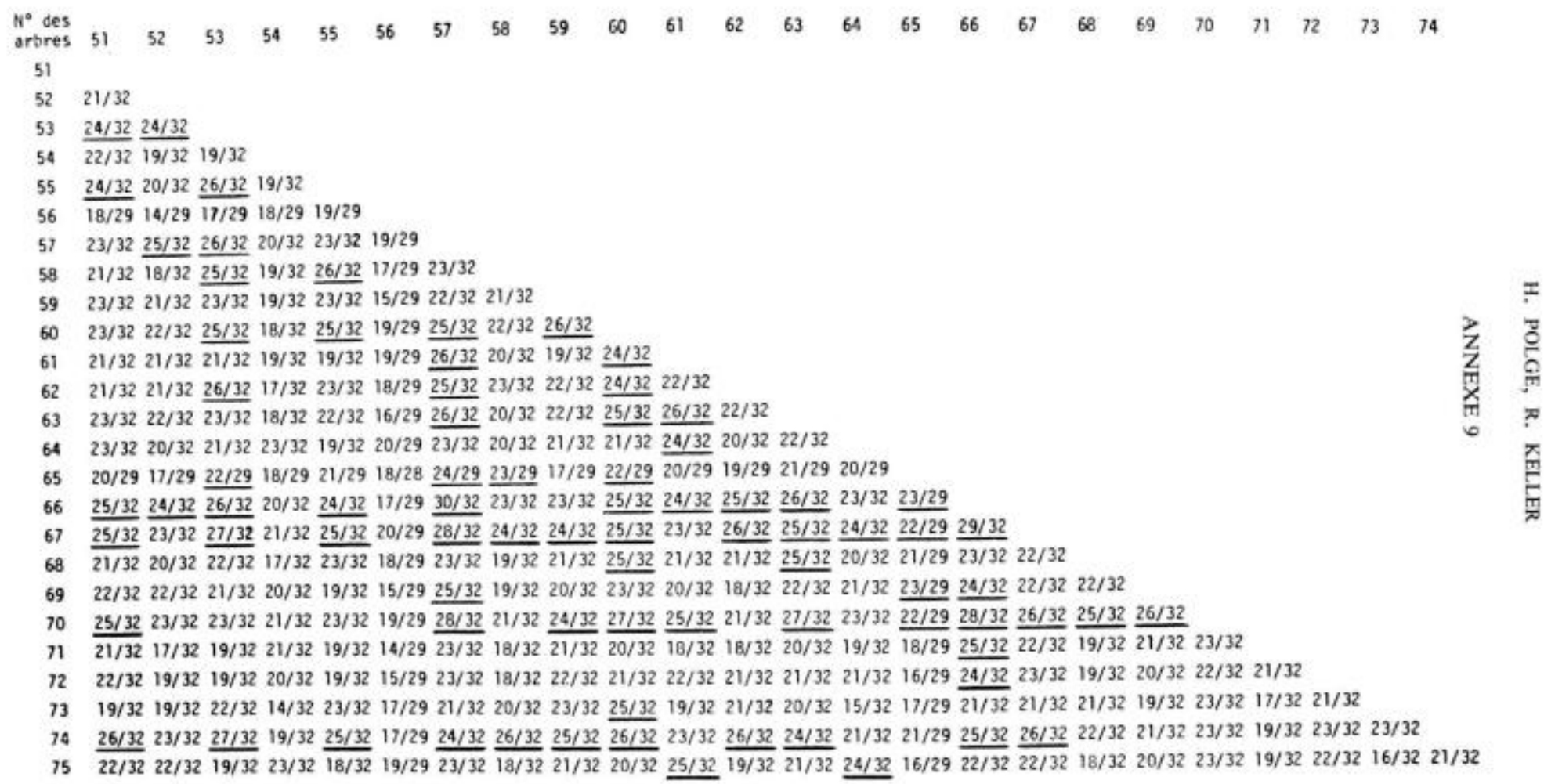

